\title{
Comparison of Bayesian Methods on Parameter Identification for a Viscoplastic Model with Damage
}

\author{
Ehsan Adeli ${ }^{1, *}$, Bojana Rosić ${ }^{2}$, Hermann G. Matthies ${ }^{3}$, Sven Reinstädler ${ }^{4}$ and Dieter Dinkler ${ }^{4}$ \\ 1 Scientific Computing and Imaging Institute, University of Utah, Salt Lake City, UT 84112, USA \\ 2 Applied Mechanics and Data Analysis, University of Twente, 7522 NB Enschede, The Netherlands; \\ wire@tu-bs.de \\ 3 Institute of Scientific Computing, Technische Universität Braunschweig, 38106 Braunschweig, Germany; \\ h.matthies@tu-bs.de \\ 4 Institute of Structural Analysis, Technische Universität Braunschweig, 38106 Braunschweig, Germany; \\ statik@tu-braunschweig.de (S.R.); d.dinkler@tu-braunschweig.de (D.D.) \\ * Correspondence: e.adeli@sci.utah.edu
}

Received: 24 May 2020; Accepted: 24 June 2020; Published: 1 July 2020

\begin{abstract}
The state of materials and accordingly the properties of structures are changing over the period of use, which may influence the reliability and quality of the structure during its life-time. Therefore, identification of the model parameters of the system is a topic which has attracted attention in the content of structural health monitoring. The parameters of a constitutive model are usually identified by minimization of the difference between model response and experimental data. However, the measurement errors and differences in the specimens lead to deviations in the determined parameters. In this article, the focus is on the identification of material parameters of a viscoplastic damaging material using a stochastic simulation technique to generate artificial data which exhibit the same stochastic behavior as experimental data. It is proposed to use Bayesian inverse methods for parameter identification and therefore the model and damage parameters are identified by applying the Transitional Markov Chain Monte Carlo Method (TMCMC) and Gauss-Markov-Kalman filter (GMKF) approach. Identified parameters by using these two Bayesian approaches are compared with the true parameters in the simulation and with each other, and the efficiency of the identification methods is discussed. The aim of this study is to observe which one of the mentioned methods is more suitable and efficient to identify the model and damage parameters of a material model, as a highly non-linear model, using a limited surface displacement measurement vector and see how much information is indeed needed to estimate the parameters accurately.
\end{abstract}

Keywords: viscoplastic-damage model; uncertainty quantification; Bayesian parameter and damage identification; functional approximation

\section{Introduction}

In order to predict the behavior of mechanically loaded metallic materials, constitutive models are applied, which present a mathematical frame for the description of elastic and inelastic deformation. All inelastic constitutive models contain parameters which have to be identified for a given material from experiments [1].

There are few investigations on the simplest material model, the elasticity model, to identify only very few parameters of the model where sampling approaches like the Metropolis algorithm and its modifications are employed. Pacheco et al. [2] investigated a three-point bending test to identify elastic behavior and calibration is done by solving the inverse problem in a Bayesian setting by using the Metropolis-Hastings algorithm. Only elastic moduli are identified without considering 
the error. Slonski et al. [3] also applied a sequential particle filter on an elastic model and Young's modulus was estimated, where the Bayesian setting was compared to the deterministic approach, and the Bayesian setting was preferred. The elastic modulus of a polymeric material is updated by Zhang et al. [4], where a Markov Chain Monte Carlo method is used, but very high computation time is reported. Further, Young's modulus is estimated for a considered material by Gallina et al. [5] by applying a multi dimensional Markov Chain Monte Carlo method. A similar Bayesian approach for composite materials to estimate Young's modulus is carried out by Pieczonka et al. [6]. Arnst et al. [7] have applied a Markov Chain Monte Carlo method by using polynomial chaos expansion to identify Young's modulus of an elastic model.

Only few investigations were carried out on enriched material models such as a viscoelastic model to identify the few parameters by employing the Metropolis-Hastings technique and the classical Markov Chain Monte Carlo method. Rappel et al. [8] studied an elastic and a viscoelastic model where the measurement error is considered. Bayesian inference is applied to estimate only the elasticity modulus by applying an adaptive Metropolis-Hastings technique. An et al. [9] investigated a crack model by a classical Markov Chain Monte Carlo method in order to estimate the parameters of a model which represent the size and position of the crack in the Bayesian setting. Also Hernandez et al. [10] applied a Markov Chain Monte Carlo method on a viscoelastic model in order to update its model parameters in a Bayesian setting, but the posterior distributions of the parameters are not updated properly. In fact, the parameters are not identified properly. Mahnken [11] has also applied a Markov Chain Monte Carlo method to estimate few parameters of a plasticity model. The damage parameters of a truss structure under model uncertainties are studied by Zheng et al. [12], where a multi-level Markov Chain Monte Carlo method is applied and the true values are not well estimated. Further, this approach suffers from high computation time. Another damage detection approach is applied by Nichols et al. [13] by applying a modified version of the Markov Chain Monte Carlo method.

There are other investigations in the Bayesian setting using the Markov Chain Monte Carlo method, and Madireddy et al [14], Wang and Zabaras [15], and Oh et al. [16] have carried out investigations on the identification of a material model by using its modified method. In studies carried out by Alvin [17], Marwala and Sibusiso [18], Daghia et al. [19], Abhinav and Manohar [20], Gogu et al. [21,22], and Koutsourelakis [23,24], the elastic parameters of the model are estimated stochastically. Fitzenz et al. [25], Most [26], and Sarkar et al. [27] investigated elastoplastic materials and thermodynamical material models to identify their model parameters. Other studies on viscoelastic models are carried out by Zhang et al. [28], Mehrez et al. [29], and Miles et al. [30]. Further investigations on viscoelastic models to estimate a higher number of model parameters are studied by Zhao and Pelegri [31], and by Kenz et al. [32]. The estimation of fatigue parameters using Markov Chain Monte Carlo methods is also studied by An et al. [33].

Few investigations in which the Kalman filter and its modifications are employed to identify the material model parameters can be found in literature. Hoshi et al. [34] have estimated the Young's Modulus and Poisson's ratio parameters of an organ model by an extended version of Kalman filter. The same study on an elastic model is carried out to identify the elastic constants of anisotropic materials using Kalman filter by Furukawa et al. [35]. Conte et al. [36] applied some modifications of Kalman filter on a nonlinear structural model to identify its parameters. Hendriks [37] investigated the possibility of identification of few parameters on a viscoelastic model representing the behavior of solid materials by using Kalman filter. Few parameters to identify on the crack path is carried out by Bolzon et al. [38] by employing Kalman filter. Nguyen et al. and Mahmoudi et al. [39-41] applied an extended Kalman filter on an elastoplastic model to identify the model parameters. Wall [42] and Nakamura et al. [43] also applied a modification of Kalman filter on a viscoplastic model to determine its model parameters probabilistically. Further, Agmell et al. [44] have determined the Johnson-Cook constitutive model constants for an orthogonal cutting process by using the Kalman filter. Sevieri et al. [45-47] investigated the parameter identification of structural models 
in the framework of structural health monitoring. Marsili et al. [48-52] investigated the update the finite element model using functional approximation of the system response.

Elastic-plastic graded materials are observed by Bocciarelli et al. [53] and Gu et al. [54] in order to identify their model parameters by employing Kalman filter. The parameters of an elastic-damage interface model are also identified by Corigliano et al. $[55,56]$ where the extended Kalman filter method is applied. Further, Ebrahimian et al. and Hariri-Ardebili et al. [57-62] investigated the damage parameter identification in the framework of structural health monitoring by using an extended version of Kalman filter. Damage detection for the purpose of health monitoring is also done by Yan et al. [63] by using the Kalman filter and other stochastic approaches by Kourehli et al. and Gharehbaghi et al. [64-66].

Although these discussed research for the identification of mechanical material models has been carried out, most of these previous research did not identify many parameters, e.g., hardening and damage parameters of the complex mechanical material models, did not quantify mostly the modeling uncertainties, did not properly deal with the ill-conditioning inherent based on the available data, and the methods employed have a high computation time even for the simplest material model considering a very few uncertain parameters. However, the uncertainty associated with the material model predictions can have a significant impact on the decision-making process in design, control, and health monitoring process.

In this paper, a viscoplastic model of Chaboche with damage is studied. The model contains five material parameters and three damage parameters which have to be determined from experimental data by using two Bayesian approaches, i.e., Transitional Markov Chain Monte Carlo method [67,68] and Gauss-Markov-Kalman filter approach [69-72]. The results of these two methods are compared and different aspects of them are evaluated. It should be noted that virtual data is employed instead of real experimental data. In addition, a cyclic tension-compression test is applied in order to extract the virtual data. A cyclic test is employed since the hardening equations as well as the damage equation could get involved so that the information from these equations can be observed.

Sections 3 and 4 explain how to propagate the uncertainty in the model. The probabilistic model is reformulated from the deterministic model, and once the forward model is provided, the model parameters are updated using two mentioned stochastic approaches.

In Section 5 the desired parameters are identified from the measured data and the efficiency of methods is studied. In fact, the parameters which have been considered as uncertain parameters are updated and their uncertainties are narrowed using Bayesian techniques. The results are thoroughly studied and the identified parameters as well as the corresponding model responses are analyzed. Finally the prediction of the models is then compared with the measured data.

\section{Model Problem}

For viscoplasticity, the dissipation potential is given by

$$
\phi_{v p}(\tilde{\boldsymbol{\sigma}})=\frac{k}{n+1}\left\langle\frac{\tilde{\sigma}_{e x}}{k}\right\rangle^{n+1} \quad \text { with } \quad \tilde{\sigma}_{e x}=\tilde{\sigma}_{e q}-\sigma_{y}-R \quad \text { and }\langle\cdot\rangle=\max (0, x)
$$

in terms of the effective equivalent stress $\tilde{\sigma}_{e q}$ and the isotropic hardening $R$ as time-varying variables. On the contrary, the yield stress $\sigma_{y}$, as well as $k$ and $n$ are further model parameters constant in time.

Based on the von-Mises yield-criterion the effective equivalent stress

$$
\tilde{\sigma}_{e q}=\sqrt{3 I_{2}(\tilde{\sigma}-\chi)^{d}+\bar{D}\left(I_{1}(\tilde{\sigma}-\chi)\right)^{2}}
$$

is described by the second Invariant

$$
I_{2}(\tilde{\sigma}-\chi)^{d}=\frac{1}{2} \operatorname{tr}\left((\tilde{\sigma}-\chi)^{d} \cdot(\tilde{\sigma}-\chi)^{d}\right)
$$


applied to the deviatoric part of the effective stress tensor $\tilde{\sigma}^{e f}=\tilde{\sigma}-\chi$ incorporating the kinematic hardening tensor $\chi$. The second term in Equation (2) takes into account that the elastic capacity is reduced when the material is damaged. Note that the first invariant

$$
I_{1}(\tilde{\sigma}-\chi)=\frac{1}{3} \operatorname{tr}(\tilde{\sigma}-\chi)
$$

is equivalent to the hydrostatic stress. The partial derivative of the dissipation potential $\phi_{v p}$ with respect to $\tilde{\sigma}$ leads to the equation for the effective viscoplastic strain

$$
\dot{\tilde{\epsilon}}_{v p}=\frac{\partial \phi_{v p}}{\partial \tilde{\sigma}}=\left\langle\frac{\tilde{\sigma}_{e x}}{k}\right\rangle^{n} \frac{\partial \tilde{\sigma}_{e x}}{\partial \tilde{\sigma}}
$$

given in rate formulation. More details are given in Appendix A. The Chaboche model allows for isotropic and kinematic hardening, which is considered in order to describe the Bauschinger effect observed at steel material under high cyclic loadings, see Simo and Hughes [73]. The evolution of the isotropic and kinematic hardening is described by the ordinary differential equations

$$
\begin{aligned}
& \dot{R}=b_{R}\left(H_{R}-R\right) \dot{p} \text { and } \\
& \dot{\chi}=b_{\chi}\left(\frac{2}{3} H_{\chi} \frac{\partial \tilde{\sigma}_{e q}}{\partial \tilde{\sigma}}-\chi\right) \dot{p}
\end{aligned}
$$

respectively, depending on the rate of accumulated equivalent viscoplastic strain

$$
\dot{p}=\left\langle\frac{\tilde{\sigma}_{e x}}{k}\right\rangle^{n}
$$

using the McAuley bracket. The parameter $b_{R}$ affects the speed of evolution, whereas the value of the parameter $H_{R}$ is the threshold for the isotropic hardening. With the same influence and meaning, the parameters $b_{\chi}$ and $H_{\chi}$ control the kinematic hardening.

The evolution equation for the local damage proposed by Kowalsky et al. [74] reads:

$$
\dot{D}=\left(c_{1}+c_{2} e^{-c_{3} p^{+}}\right) \dot{p}^{+}+c_{4}\left(c_{5}-\bar{D}\right)\left\langle\operatorname{tr}\left(\frac{\partial \tilde{\sigma}_{e q}}{\partial \tilde{\sigma}} \dot{p}^{+}\right)\right\rangle .
$$

It is regularized by the model parameter $c_{1}$ up to $c_{5}$ and only activated if the threshold $\epsilon_{v p}^{e q}$ for the viscoplastic strain is reached, thus $\sqrt{2 / 3 \epsilon_{v p}: \epsilon_{v p}}>\epsilon_{v p}^{e q}$. Achieving a model where damage only accumulates with positive hydrostatic stress, Equation (8) is formulated by the active accumulated plastic strain $p^{+}$, an internal variable increasing with

$$
\dot{p}^{+}=\dot{p}\left\langle\frac{I_{1}}{\left|I_{1}\right|}\right\rangle,
$$

see Pirondi and Bon [75]. For the parameter identification, the initial conditions $R(0)=0, \chi(0)=\mathbf{0}$ and $D(0)=0$ are assumed. The complete material model is summarized in Table 1.

The governing equations above are numerically solved using the space-time finite element method (ST-FEM), see Hughes and Hulbert [76]. The ST-FEM is the consistent extension of the finite element method [77] with a time integration scheme following the Galerkin method, thus both, in space and time, the differential equations of the structure are approximately solved with the same numerical method. 
By gathering all the desired material parameters to identify into the vector $\boldsymbol{q}=\left[\kappa G b_{R} b_{\chi} \sigma_{y} c_{1} c_{2} c_{3}\right]$, where $\kappa$ and $G$ are bulk modulus and shear modulus respectively, the goal is to estimate $q$ given measurement displacement data, i.e.,

$$
u=Y(q)+\varepsilon
$$

in which $Y(\boldsymbol{q})$ represents the measurement operator and $\varepsilon$ the measurement (also possibly the model) error. Being an ill-posed problem, the estimation of $q$ given $u$ is not an easy task and requires regularization. This can be achieved either in a deterministic or probabilistic setting. Here, the latter one is taken into consideration as further described in the text.

Table 1. Chaboche-type material with hardening and damage.

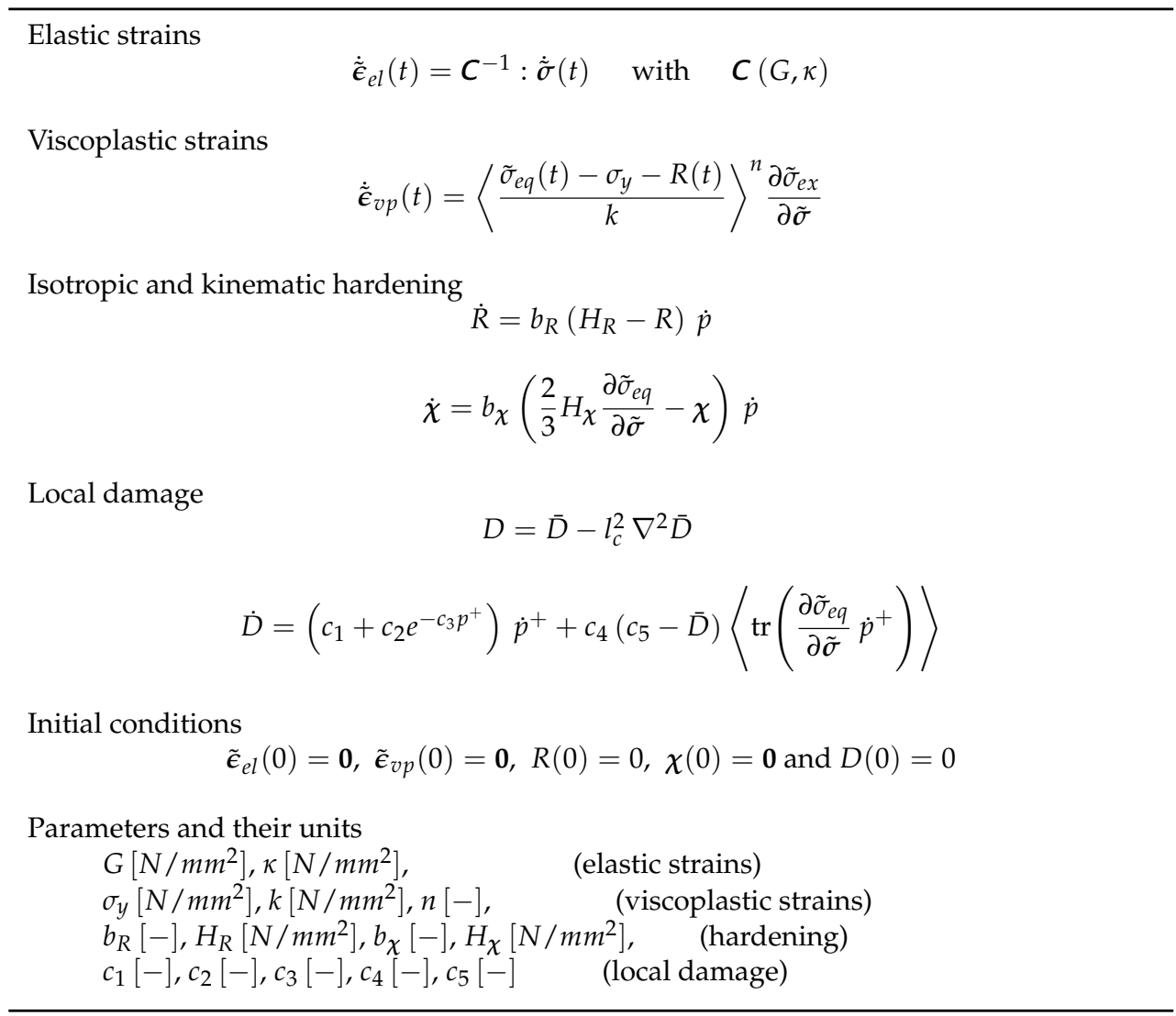

\section{Bayesian Identification}

The main methods are applied on the case study and their efficiencies will be studied in Section 5 . The first method is the so-called Transitional Markov chain Monte Carlo method and the other one is the Gauss-Markov-Kalman filter which are presented in the following.

\subsection{Transitional Markov Chain Monte Carlo Method}

The Transitional Markov Chain Monte Carlo (TMCMC) method is proposed by Ching and Chen $[67,68]$. The generation of samples from the posterior PDF can be a difficult task due to the lack of information about the geometry of the probability density function and in order to overcome this problem, the TMCMC algorithm uses a sequence of intermediate PDFs that converge to the target posterior PDF, which are defined by:

$$
f_{j}(\boldsymbol{\theta}) \propto f(\boldsymbol{\theta} \mid \mathcal{M}) f(\mathcal{D} \mid \mathcal{M}, \boldsymbol{\theta})^{r_{j}}
$$


where $j=0,1,2, \ldots, M$ and $0=r_{0} \leq r_{1} \leq r_{2} \leq \ldots \leq r_{M}=1$ in which the index $j$ denotes the stage number. As one can see, this construction fulfills the desirable properties: the series of intermediate PDFs starts from the prior PDF, i.e., $r_{j}=0$, and ends with $f(\boldsymbol{\theta} \mid \mathcal{M}, \mathcal{D})$, i.e., $r_{j}=1$, by considering that $f_{0}(\boldsymbol{\theta})=f(\boldsymbol{\theta} \mid \mathcal{M})$ and $f_{M}(\boldsymbol{\theta})=f(\boldsymbol{\theta} \mid \mathcal{M}, \mathcal{D})$, which means:

$$
\begin{array}{cc}
r_{j}=0 & f_{0}(\boldsymbol{\theta}) \propto f(\boldsymbol{\theta} \mid \mathcal{M}) \\
r_{j}=1 & f_{M}(\boldsymbol{\theta}) \propto f(\boldsymbol{\theta} \mid \mathcal{M}, \mathcal{D})
\end{array}
$$

The idea is that although the geometry change from $f(\boldsymbol{\theta} \mid \mathcal{M})$ to $f(\boldsymbol{\theta} \mid \mathcal{M}, \mathcal{D})$ can be dramatic, the change between two adjacent intermediate PDFs can be small. This small transition makes it possible to obtain efficiently samples from $f_{j+1}(\boldsymbol{\theta})$ based on samples from $f_{j}(\boldsymbol{\theta})$.

\section{Transitional MCMC Algorithm}

The idea behind TMCMC is described in detail in this subsection. The entire algorithm is presented in the following $[67,68,78]$.

1. In the first step, $j=0$ is set and from $f_{0}(\boldsymbol{\theta})=f(\boldsymbol{\theta} \mid \mathcal{M})$ a set of $N$ samples are picked up.

2. $r_{j+1}$ should be selected in a way that the coefficient of variation of $f\left(\mathcal{D} \mid \mathcal{M}, \boldsymbol{\theta}_{k}^{(j)}\right)^{r_{j+1}-r_{j}}$ where $k=1,2, \ldots, N$ is equal to a prescribed value. Accordingly, the coefficient of variation serves as an indicator of how close is $f_{j}(\boldsymbol{\theta})$ to $f_{j+1}(\boldsymbol{\theta})$.

3. The plausibility weight $w\left(\boldsymbol{\theta}_{k}^{(j)}\right)=f\left(\mathcal{D} \mid \mathcal{M}, \boldsymbol{\theta}_{k}^{(j)}\right)^{r_{j+1}-r_{j}}$ is calculated for $k=1,2, \ldots, N$ and the parameter $S^{j}=\sum_{k=1}^{N} w\left(\boldsymbol{\theta}_{k}^{(j)}\right) / N^{j}$ is computed.

4. Samples $\boldsymbol{\theta}_{k}^{j+1}$ for $k=1,2, \ldots, N$ are provided from $f(\boldsymbol{\theta})^{j+1}$ by Metropolis-Hastings algorithm. In other words, the $k$-th sample is picked up from a Markov Chain that starts from one of the samples $\boldsymbol{\theta}_{i}^{j}$ where $i=1,2, \ldots, N$ which is selected randomly. The $i$-th initial sample $\boldsymbol{\theta}_{i}^{(j)}$ is chosen with probability $w\left(\boldsymbol{\theta}_{i}^{(j)}\right) / \sum_{l=1}^{N} w\left(\boldsymbol{\theta}_{l}^{(j)}\right)$. As proposal distribution, a Gaussian which is centered at the current sample in the k-th chain is applied in Metropolis-Hastings algorithm. Also, the covariance matrix:

$$
\boldsymbol{\Sigma}_{j}=\beta^{2} \frac{\sum_{i=1}^{N} w\left(\boldsymbol{\theta}_{i}^{(j)}\right)\left[\boldsymbol{\theta}_{i}^{(j)}-\boldsymbol{\mu}_{j}\right]\left[\boldsymbol{\theta}_{i}^{(j)}-\boldsymbol{\mu}_{j}\right]^{T}}{\sum_{k=1}^{N} w\left(\boldsymbol{\theta}_{k}^{(j)}\right)}
$$

where

$$
\boldsymbol{\mu}_{j}=\frac{\sum_{l=1}^{N} w\left(\boldsymbol{\theta}_{l}^{(j)}\right) \boldsymbol{\theta}_{l}^{(j)}}{\sum_{l=1}^{N} w\left(\boldsymbol{\theta}_{l}^{(j)}\right)}
$$

in which $\beta$ is a prescribed scaling factor which is used as the rejection rate in MetropolisHastings algorithm.

5. Steps two to four are repeated until $r_{M}=1$. At the final step, samples $\boldsymbol{\theta}_{k}^{(M)}$ for $k=1,2, \ldots, N$ are distributed according to $f(\boldsymbol{\theta} \mid \mathcal{M}, \mathcal{D})$ and it is turned out that $S=\Pi_{j=0}^{M} S_{j}$ is asymptotically unbiased for $f(\mathcal{D} \mid \mathcal{M})$.

One of the significant advantages of modified Metropolis-Hastings, which is applied in TMCMC, is that the first levels allow a free exploring of the sample space, while, in the last simulation levels, the sampling is performed from a narrower neighborhood of the sample space. Furthermore, the proposal distribution changes within the same simulation level and this property causes a better local behavior. This is accomplished by modifying the proposal distribution for each level, in such a 
manner that its standard deviation is small for higher simulation levels, while the average value drives the sampling towards the most important neighborhood of the sample space $[67,68,78]$.

\subsection{Gauss-Markov-Kalman Filter Using Functional Approximation}

Some methods used for estimation of Bayes's theorem unlike Monte Carlo methods do not use all information but only part of information as approximations are considered. Hence the balance between time consumption, considered amount of information and the accuracy of approximations can be achieved. Incidentally, this leads to Kalman filter (KF) [79-81] method as it was related to Gauss-Markov theorem which is developed without any reference to Bayes's theorem. Moreover, the polynomial chaos expansion is also used along with the ensemble Kalman filter (EnKF) $[69,82]$ in order to be completely independent from any time consuming computational implementations such as Monte Carlo method. This leads to the Gauss-Markov-Kalman filter [69-72] which is discussed in this section and more details are provided in Appendix B.

\subsubsection{The Linear Filter}

The minimization problem as in the Equation (A26) is to be solved and it lies in infinite dimensional space. Hence it is to be approximated using Galerkin method in finite dimensional subspaces. The chosen desired subspace to solve the problem is $\mathscr{Q}_{1} \subset \mathscr{Q}_{\infty} \subset \mathscr{Q}$. Hence the desired subspace is shown in Equation (16) where the affine maps $\Phi$ are certainly measurable [69-71,83].

$$
\mathscr{Q}_{1}=\{z: z=\Phi(y)=L(y(\omega))+b, L \in \mathscr{L}(\mathcal{Y}, \mathcal{Q}), b \in \mathcal{Q}\} \subset \mathscr{Q}_{\infty} \subset \mathscr{Q}
$$

The Equation (17) is obtained from the minimization problem as given by the Equation (A26) where the optimal affine map is introduced via so-called Kalman gain $K \in \mathscr{L}(\mathcal{Y}, \mathcal{Q})$. The Kalman gain is represented as $K:=\operatorname{cov}(\boldsymbol{q}, y) \operatorname{cov}(y)^{-1}$ where $\operatorname{cov}(\boldsymbol{q}, y)$ is the covariance of $\boldsymbol{q}$ and $y, \operatorname{cov}(y)$ is the auto-covariance of $y$ and $a \in \mathcal{Q}$ read as $a:=\bar{q}-K(\bar{y})$.

$$
\|\boldsymbol{q}-(\boldsymbol{K}(y)+a)\|_{\mathscr{Q}}^{2}=\min _{L, b}\|\boldsymbol{q}-(L(y)+b)\|_{\mathscr{Q}}^{2}
$$

It should be noted that as $\mathscr{Q}_{1} \subset \mathscr{Q}_{\infty}$ is a true subspace, then obviously some information is disregarded when using this approximation $g(y)=K(y)+a$. Although the computation becomes easier, some information that we may learn from the measurement is neglected. Equation (18) is determined from the Equation (A23) and from the described algorithm.

$$
\boldsymbol{q}_{a, 1 L}=\boldsymbol{q}_{f}+(\boldsymbol{K}(\hat{y})-\boldsymbol{K}(y))=\boldsymbol{q}_{f}+\boldsymbol{K}(\hat{y}-y)
$$

This linear filter is called Gauss-Markov-Kalman filter (GMKF) with the linear minimum mean square error $\boldsymbol{K}(\hat{y})$ defined as in [69-72]. It should be noted that Gauss-Markov-Kalman filter is a general form of the original Kalman filter which is considered only for the mean values of the random variables of the parameters included in Equation (18). Accordingly the Equation (A25) representing the algorithm turns to the Equation (19).

$$
\left.\boldsymbol{q}_{n+1}=\boldsymbol{q}_{n}+\boldsymbol{K}\left(\left(\hat{y}_{n+1}\right)-\left(Y\left(\boldsymbol{q}_{n}\right)+\epsilon\right)\right)=\boldsymbol{q}_{n}-\boldsymbol{K}\left(Y\left(\boldsymbol{q}_{n}\right)+\epsilon\right)\right)+\boldsymbol{K}\left(\hat{y}_{n+1}\right)
$$

Equation (20) is determined from the Equation (18) by introducing the Kalman gain and by considering the random variables as the argument. By considering the error the Kalman gain is defined as $\boldsymbol{K}:=\operatorname{cov}\left(\boldsymbol{q}_{f}, y\right)(\operatorname{cov}(y)+\operatorname{cov}(\epsilon))^{\dagger}$.

$$
\boldsymbol{q}_{a}(\omega)=\boldsymbol{q}_{f}(\omega)+\boldsymbol{K}(\hat{y}-y(\omega))
$$


The Gauss-Markov-Kalman filter as described in Equation (20) needs to be discretized in order to implement numerically as it is related with the random variables.

\subsubsection{Sequential Gauss-Markov-Kalman Filter}

The process of updating can be done several times on the whole time interval. Once a high non-linear chaotic system is divided to very small time steps, the problem turns to plenty of continuous linear systems so that the GMKF approach can update the model parameters in a much better way if it is applied several times to update the model parameters on each time step. Eventually this approach helps to update the uncertain parameters of a non-linear system. Therefore the Gauss-Markov-Kalman filter as in Equation (20) can be written as like in Equation (21) on the k-th time step for the $n$ total time steps, where the Kalman gain reads $K^{(k)}:=\operatorname{cov}\left(\boldsymbol{q}_{f}^{(k)}, y^{(k)}\right)\left(\operatorname{cov}\left(y^{(k)}\right)+\operatorname{cov}(\epsilon)\right)^{\dagger}$.

$$
\boldsymbol{q}_{a}^{(k)}(\omega)=\boldsymbol{q}_{a}^{(k-1)}(\omega)+\boldsymbol{K}^{(k)}\left(\hat{y}^{(k)}-y^{(k)}(\omega)\right)
$$

The Equation (21) is called sequential Gauss-Markov-Kalman filter. Schematically the process of sequential Gauss-Markov-Kalman filter is shown in Figure 1 where the posterior of one update is the prior of the next update.

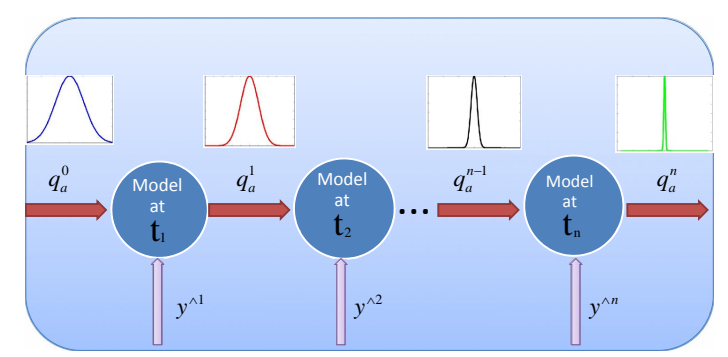

Figure 1. Sequential GMKF method.

The approaches such as sampling and spectral approximation or functional approximations to compute the linear filter approximations are discussed in this section.

\subsubsection{Sampling}

Considering $N$ random variables an ensemble of sampling points $\omega=\left[\omega_{1}, \ldots, \omega_{N}\right]$ are taken into account [82]. The Gauss-Markov-Kalman filter as shown in Equation (20) for the considered ensemble of sampling points results in Equation (22).

$$
\forall \ell=1, \ldots, N: \boldsymbol{q}_{a}\left(\omega_{\ell}\right)=\boldsymbol{q}_{f}\left(\omega_{\ell}\right)+\boldsymbol{C}_{\boldsymbol{q}_{f} y}\left(\boldsymbol{C}_{y}+\boldsymbol{C}_{\epsilon}\right)^{\dagger}\left(\check{y}-y\left(\omega_{\ell}\right)\right)
$$

The Equation (22) is the basis of ensemble Gauss-Markov-Kalman filter where $\boldsymbol{C}_{\boldsymbol{q}_{f} y}=\operatorname{cov}\left(\boldsymbol{q}_{f}, y\right)$, $C_{y}=\operatorname{cov}(y)$ and $C_{\epsilon}=\operatorname{cov}(\epsilon) \cdot \boldsymbol{q}_{f}\left(\omega_{l}\right)$ and $\boldsymbol{q}_{a}\left(\omega_{l}\right)$ are shown as particles in the extended version.

\subsubsection{Functional Approximation}

The discretization of the random variables are performed by spectral or functional approximations instead of sampling $[70,84,85]$. Hence the desired random variables are described as functions of known random variables $\left\{\theta_{1}(\omega), \ldots, \theta_{l}(\omega), \ldots\right\}$. As only finite random variables can be dealt, a finite vector random variables in functional representation $\boldsymbol{\theta}(\omega)=\left[\theta_{1}(\omega), \ldots, \theta_{n}(\omega)\right]$ can be considered where $n$ random variables $\boldsymbol{\theta}$ are taken into account.

The polynomial chaos expansion [86,87] is chosen as system of functions but also other possibilities exist. It should be noted that the finite set of linear independent Hermite functions $\left\{H_{\alpha}\right\}_{\alpha \in \mathcal{J}_{M}}$ of variables $\boldsymbol{\theta}(\omega)$ should include all the linear functions of $\boldsymbol{\theta}$ with polynomials such as polynomial chaos 
expansion [69-71]. The multi-index is represented by $\alpha$ and the set $\mathcal{J}_{M}$ is a finite set with cardinality $M$. The functional approximation of a random variable $\boldsymbol{q}(\omega)$ is shown in Equation (23).

$$
\boldsymbol{q}(\omega)=\sum_{\alpha \in \mathcal{J}_{M}} \boldsymbol{q}_{\alpha} H_{\alpha}(\boldsymbol{\theta}(\omega))=\sum_{\alpha \in \mathcal{J}_{M}} \boldsymbol{q}_{\alpha} H_{\alpha}(\boldsymbol{\theta})=\boldsymbol{q}(\boldsymbol{\theta})
$$

The argument $\omega$ is neglected in the Equation (23) because the probability measure $\mathbb{P}$ on $\Omega$ is transported to $\Theta=\Theta_{1} \times \ldots \times \Theta_{n}$. The range of $\boldsymbol{\theta}$ showing $\mathbb{P}_{\theta}=\mathbb{P}_{1} \times \ldots \times \mathbb{P}_{n}$ as a product measure, where $\mathbb{P}_{\ell}=\left(\theta_{\ell}\right)_{*} \mathbb{P}$ is the distribution measure of the random variable $\theta_{\ell}$ and it is noted that the random variables $\theta_{\ell}$ are independent. Therefore all computations are performed on $\Theta$ which is typically a subset of $\mathbb{R}^{n}$. Hence the Gauss-Markov-Kalman filter as shown in Equation (20) for the considered expansion results in Equation (24), with the indicated inverse sign $t$, which is known as spectral Gauss-Markov-Kalman filter.

$$
\boldsymbol{q}_{a}(\boldsymbol{\theta})=\boldsymbol{q}_{f}(\boldsymbol{\theta})+\boldsymbol{C}_{\boldsymbol{q}_{f} y}\left(\boldsymbol{C}_{y}+\boldsymbol{C}_{\epsilon}\right)^{\dagger}(\check{y}-y(\boldsymbol{\theta}))=\boldsymbol{q}_{f}(\boldsymbol{\theta})+\boldsymbol{K}(\check{y}-y(\boldsymbol{\theta}))
$$

It should also be noted that in spectral approximation the Gauss-Markov-Kalman filter as shown in the Equation (20) has the same form as of the sampling approach but the only difference is the functional approximation of random variables, i.e., the Hermite functions are used to calculate the covariance matrices. For instance $\boldsymbol{C}_{\boldsymbol{q}_{f} y}$ can be easily computed as given in Equation (25).

$$
\boldsymbol{C}_{\boldsymbol{q}_{f} y}=\sum_{\alpha>0} \alpha !\left(\boldsymbol{q}_{f}^{(\alpha)}(\boldsymbol{\theta})\right)\left(y^{(\alpha)}(\boldsymbol{\theta})\right)^{T}
$$

While the Equation (20) is applied on samples or particles in sampling approach, in spectral approximations it is applied on the coefficients as shown in Equation (23) which are the functional approximation of the random variables.

\section{Numerical Results}

The identification of the material constants in the Chaboche unified viscoplasticity model with damage is a reverse process based on virtual data. The aim of the parameter estimation is to find a parameter vector $\boldsymbol{q}$ introduced in the previous section. The bulk modulus $(\kappa)$, the shear modulus $(G)$, the isotropic hardening coefficient $\left(b_{R}\right)$, the kinematic hardening coefficient $\left(b_{\chi}\right)$ and the yield stress $\left(\sigma_{y}\right)$ as well as damage parameters $\left(c_{1}, c_{2}\right.$ and $\left.c_{3}\right)$ are considered as uncertain parameters of the constitutive model.

As both kinds of hardenings are taken into account and the studied model is a damaged material model, one appropriate way of the identification of parameters is to use the results of the cyclic tests as observation, since more information can be obtained from virtual data rather than from creep and relaxation tests, especially information regarding hardening and damage parameters as the hardening and damage equations are involved in this case.

Two tests are studied to observe the efficiency of the identified parameters using different Bayesian approaches which are discussed in the following.

Validation Procedure for Viscoplasticity Model with Isotropic and Kinematic Hardening Behavior

The Transitional Markov Chain Monte Carlo method and Gauss-Markov-Kalman filter using the polynomial chaos expansion are applied on a viscoplastic model considering both the isotropic and kinematic hardening behavior.

The parameters which are set as the uncertain parameters are bulk modulus $(\kappa)$, shear modulus $(G)$, the isotropic hardening coefficient $\left(b_{R}\right)$, the kinematic hardening coefficient $\left(b_{\chi}\right)$ and the yield stress $\left(\sigma_{y}\right)$. Hence the vector of unknown parameters is $\boldsymbol{q}=\left[\kappa(\omega), G(\omega), b_{R}(\omega), b_{\chi}(\omega), \sigma_{y}(\omega)\right]$.

A preliminary study in the following of our previous studies [88-90] is on a regular cube, modeled with one 8 node element. The minimal number of freedoms that have to be constrained is six and 
many combinations are possible. As shown in Figure 2, all three degrees of freedom at point B are fixed. This prevents all rigid body translations, and leaves three rotations to be taken care of. The $x$ displacement component at point $\mathrm{A}$ is constrained to prevent rotation about $z$, and the $z$ component is fixed at point $C$ to prevent rotation about $y$. The $y$ component is constrained at point $D$ to prevent rotation about $x$ [91].

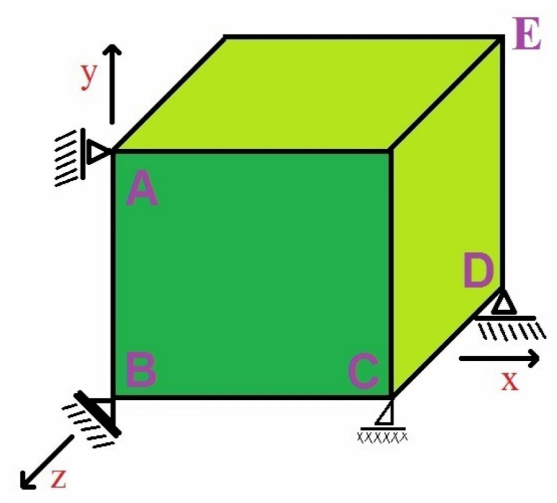

Figure 2. Boundary condition considered.

The normal tractions, which is a Neumann boundary condition are applied cyclically in $x, y$ and $z$ directions on front and back faces and the magnitude of tractions in all directions are shown in Figure 3 where green, red and blue colors represent the stress values in $x, y$ and $z$ directions respectively.

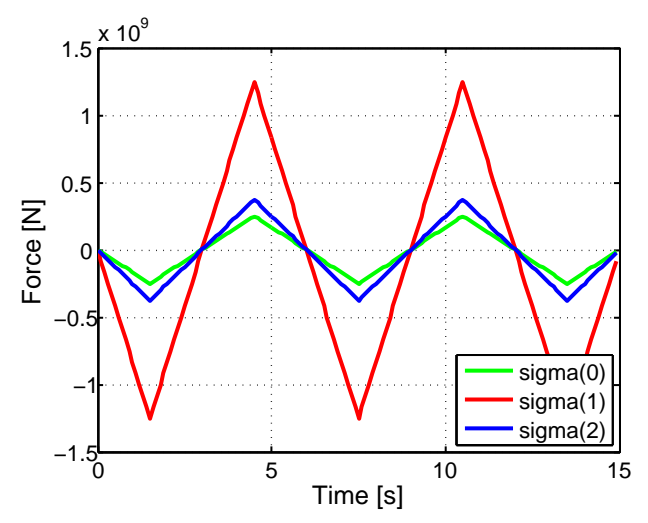

Figure 3. Decomposed applied force at point E according to time.

By considering the parameters listed in Table 2, for the top right corner node on back face, point E, as shown in Figure 2, the related displacement graph is obtained as shown in Figure 4 where green, red and blue colors represent the displacement of point $\mathrm{E}$ in $x, y$ and $z$ directions respectively.

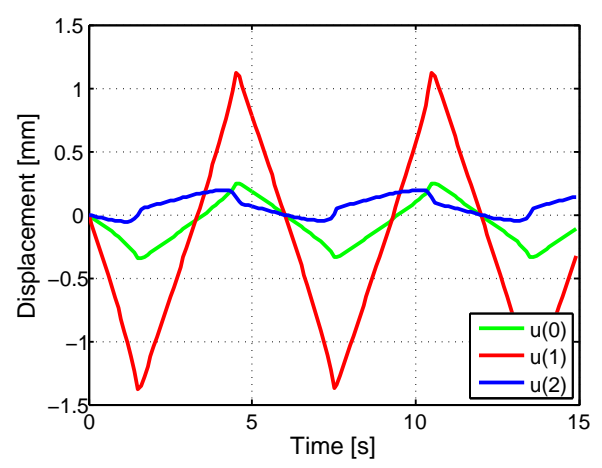

Figure 4. Displacement of point $\mathrm{E}$ in $x, y$ and $z$ directions according to time. 
Table 2. The model parameters.

\begin{tabular}{lcccccccc}
\hline $\boldsymbol{\kappa}$ & $\boldsymbol{G}$ & $\sigma_{y}$ & $\boldsymbol{n}$ & $\boldsymbol{k}$ & $\boldsymbol{b}_{\boldsymbol{R}}$ & $\boldsymbol{H}_{\boldsymbol{R}}$ & $\boldsymbol{b}_{\chi}$ & $\boldsymbol{H}_{\chi}$ \\
\hline $1.66 \times 10^{9}$ & $7.69 \times 10^{8}$ & $1.7 \times 10^{8}$ & 1 & $1.5 \times 10^{8}$ & 50 & $2.75 \times 10^{8}$ & 50 & $2.75 \times 10^{8}$ \\
\hline
\end{tabular}

The displacements of point $\mathrm{E}$ in $x, y$ and $z$ directions are noted as the virtual data in this case.

Applying both the stochastic identifications, which are fully discussed in Sections 3.1 and 3.2, i.e., Transitional Markov Chain monte Carlo method and history matching Gauss-Markov-Kalman filter and sequential Gauss-Markov-Kalman filter approach, the probability density functions of prior and posterior of the identified parameters can be seen and compared in Figure 5. It should be pointed out that in the history matching updating the whole time interval is considered and update is done only once by comparing the predicted value with the recorded displacement history. On the other hand in the sequential updating the process of updating is done several times.

Summarizing the results, the true values and the mean and variance of the estimated parameters for each Bayesian method are compared in Table 3.

Table 3. The identified model parameters.

\begin{tabular}{lccccccc}
\hline Param. & $\boldsymbol{q}_{\text {true }}$ & $\boldsymbol{q}_{\text {TMCMC }}^{m}$ & $\boldsymbol{q}_{\text {TMCMC }}^{\text {std }}$ & $\boldsymbol{q}_{\text {GMKF }}^{m}$ & $\boldsymbol{q}_{\text {GMKF }}^{\text {std }}$ & $\boldsymbol{q}_{\text {SGMKF }}^{m}$ & $\boldsymbol{q}_{\text {SGMKF }}^{\text {std }}$ \\
\hline$\kappa$ & $1.66 \times 10^{9}$ & $1.71 \times 10^{9}$ & $1.33 \times 10^{7}$ & $1.66 \times 10^{9}$ & $2.28 \times 10^{7}$ & $1.66 \times 10^{9}$ & $1.13 \times 10^{7}$ \\
$G$ & $7.69 \times 10^{8}$ & $7.66 \times 10^{8}$ & $2.24 \times 10^{6}$ & $7.68 \times 10^{8}$ & $9.09 \times 10^{6}$ & $7.68 \times 10^{8}$ & $3.47 \times 10^{6}$ \\
$b_{R}$ & 50 & 53.00 & 1.83 & 54.78 & 5.16 & 52.36 & 3.71 \\
$b_{\chi}$ & 50 & 49.30 & 1.32 & 54.58 & 5.15 & 52.04 & 3.01 \\
$\sigma_{y}$ & $1.7 \times 10^{8}$ & $1.67 \times 10^{8}$ & $1.37 \times 10^{6}$ & $1.67 \times 10^{8}$ & $3.48 \times 10^{6}$ & $1.69 \times 10^{8}$ & $1.35 \times 10^{6}$ \\
\hline
\end{tabular}
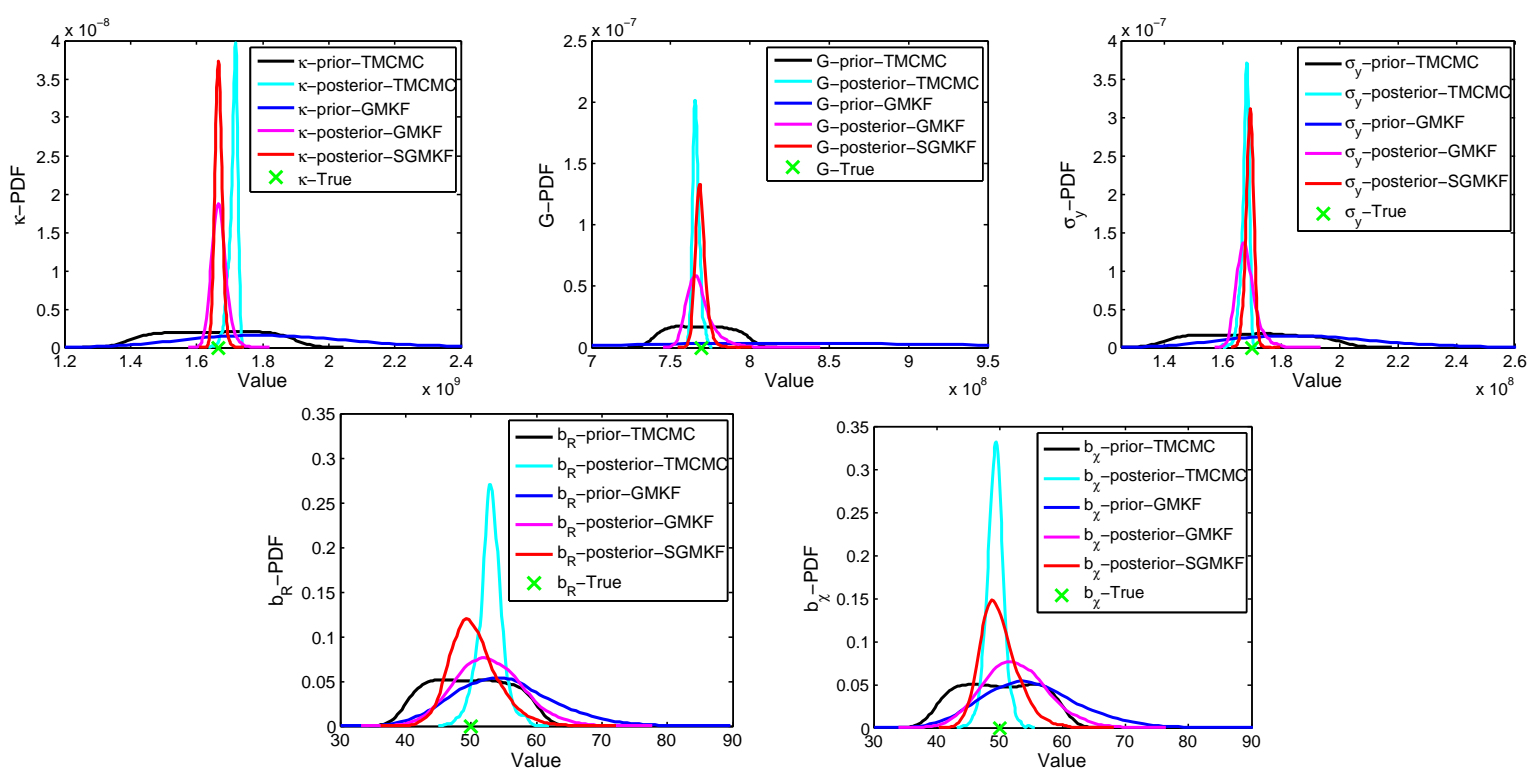

Figure 5. PDF of identified parameters.

\section{Discussion and Comparison}

By comparing the results from the two main approaches, it can be concluded Transitional Markov Chain Monte Carlo and Gauss-Markov-Kalman filter method provide accurate result and identify the known parameters for the viscoplasticity model with isotropic and kinematic hardening behavior except in the history matching update approach as the Gauss-Markov-Kalman filter method for the hardening parameters are not as narrow as the rest of parameter's distributions as shown in Figure 5. This is because the hardening parameters have no direct and strong influence on the measured 
displacement and so the uncertainties are not reduced good enough than rest of the parameters as there is no enough information. As another reason it can be mentioned that updating is done only once when history matching Gauss-Markov-Kalman filter is applied and hence the posterior distributions of hardening parameters are not as narrow as when sequential Gauss-Markov-Kalman filter is employed.

In addition, as the computations are evaluated from the initial time step after each update in the sequential Gauss-Markov-Kalman filter, more information is recorded in total. Therefore, better identification and reduction of uncertainty can be obtained in comparison with history matching Gauss-Markov-Kalman filter.

There is not much difference between the standard deviation of the uncertain parameters between Transitional Markov Chain Monte Carlo approach and Gauss-Markov-Kalman filter approach for sequential updating of viscoplasticity with isotropic and kinematic hardening behavior. It should also be noted that the Transitional Markov Chain Monte Carlo method computational time is significantly higher than the other approach. The computational time is more in the Gauss-Markov-Kalman filter approach as multiple updating is involved in the sequential updating of Gauss-Markov-Kalman filter approach.

As Transitional Markov Chain Monte Carlo approach applied on viscoplasticity model with isotropic and kinematic hardening is computationally too much expensive, Gauss-Markov-Kalman filter method is only considered for the viscoplastic-damage model in Section 5.1.

\subsection{Validation Procedure on Viscoplastic-Damage Model with Isotropic and Kinematic Hardening}

In this section the capability of only one of the Bayesian approaches discussed in the previous sections is studied. Gauss-Markov-Kalman filter using the polynomial chaos expansion is applied on a viscoplastic-damage model with both the isotropic and kinematic hardening behavior.

The parameters which are set as the uncertain parameters are bulk modulus $(\kappa)$, shear modulus $(G)$, the isotropic hardening coefficient $\left(b_{R}\right)$, the kinematic hardening coefficient $\left(b_{\chi}\right)$ and the yield stress $\left(\sigma_{y}\right)$ as well as damage parameters $\left(\left(c_{1}\right),\left(c_{2}\right)\right.$ and $\left.\left(c_{3}\right)\right)$. Hence the vector of unknown parameters is $\boldsymbol{q}=\left[\kappa(\omega), G(\omega), \sigma_{y}(\omega), b_{R}(\omega), b_{\chi}(\omega), c_{1}(\omega), c_{2}(\omega), c_{3}(\omega)\right]$.

Similar to the previous case, preliminary study is on a regular cube, modeled with one 8 node element as shown in Figure 2. Similarly the same Dirichlet boundary condition is considered. Also the same tractions which is a Neumann boundary condition are applied cyclically in $x, y$ and $z$ directions on front and back faces and the magnitude of tractions in all directions are shown in Figure 6 where green, red and blue colors represent the stress values in $x, y$ and $z$ directions respectively.

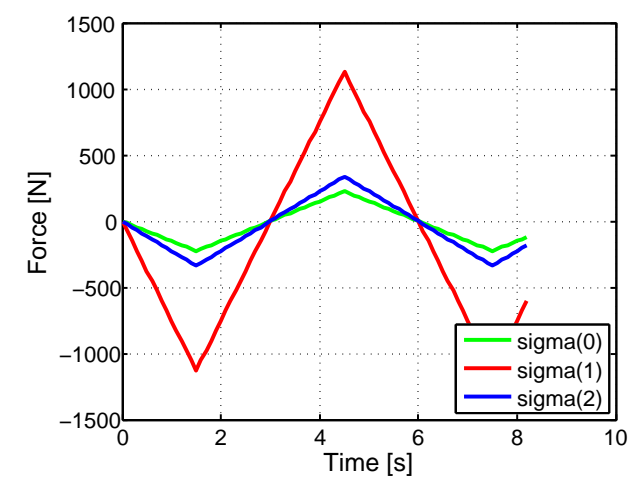

Figure 6. Decomposed applied force at point E according to time.

By considering the parameters listed in Table 4, for the top right corner node on back face, point E, as shown in Figure 2, the corresponding displacement graph is obtained as shown in Figure 7 where green, red and blue colors represent the displacement of point $\mathrm{E}$ in $x, y$ and $z$ directions respectively. 
Table 4. The model parameters.

\begin{tabular}{lccccccccccc}
\hline $\boldsymbol{\kappa}$ & $\boldsymbol{G}$ & $\sigma_{\boldsymbol{y}}$ & $\boldsymbol{n}$ & $\boldsymbol{k}$ & $\boldsymbol{b}_{\boldsymbol{R}}$ & $\boldsymbol{H}_{\boldsymbol{R}}$ & $\boldsymbol{b}_{\chi}$ & $\boldsymbol{H}_{\chi}$ & $\boldsymbol{c}_{\boldsymbol{1}}$ & $\boldsymbol{c}_{\mathbf{2}}$ & $\boldsymbol{c}_{\boldsymbol{3}}$ \\
\hline $1.66 \times 10^{5}$ & $7.69 \times 10^{4}$ & 266 & 1 & 23,500 & 298.6 & 117.2 & 100 & 150 & 7 & 8 & -80 \\
\hline
\end{tabular}

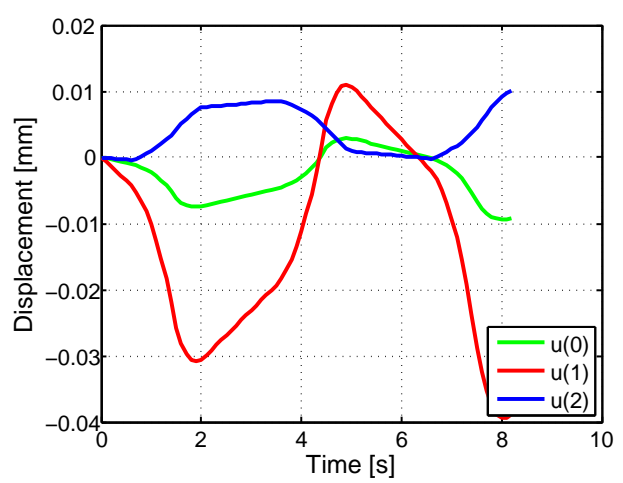

Figure 7. Displacement of point $\mathrm{E}$ in $x, y$ and $z$ directions according to time.

Again the displacements of point $\mathrm{E}$ in $x, y$ and $z$ directions are noted as the virtual data in this case. Applying the Gauss-Markov-Kalman filter approach, the probability density functions of prior and posterior of the identified parameters can be seen and compared in Figure 8.
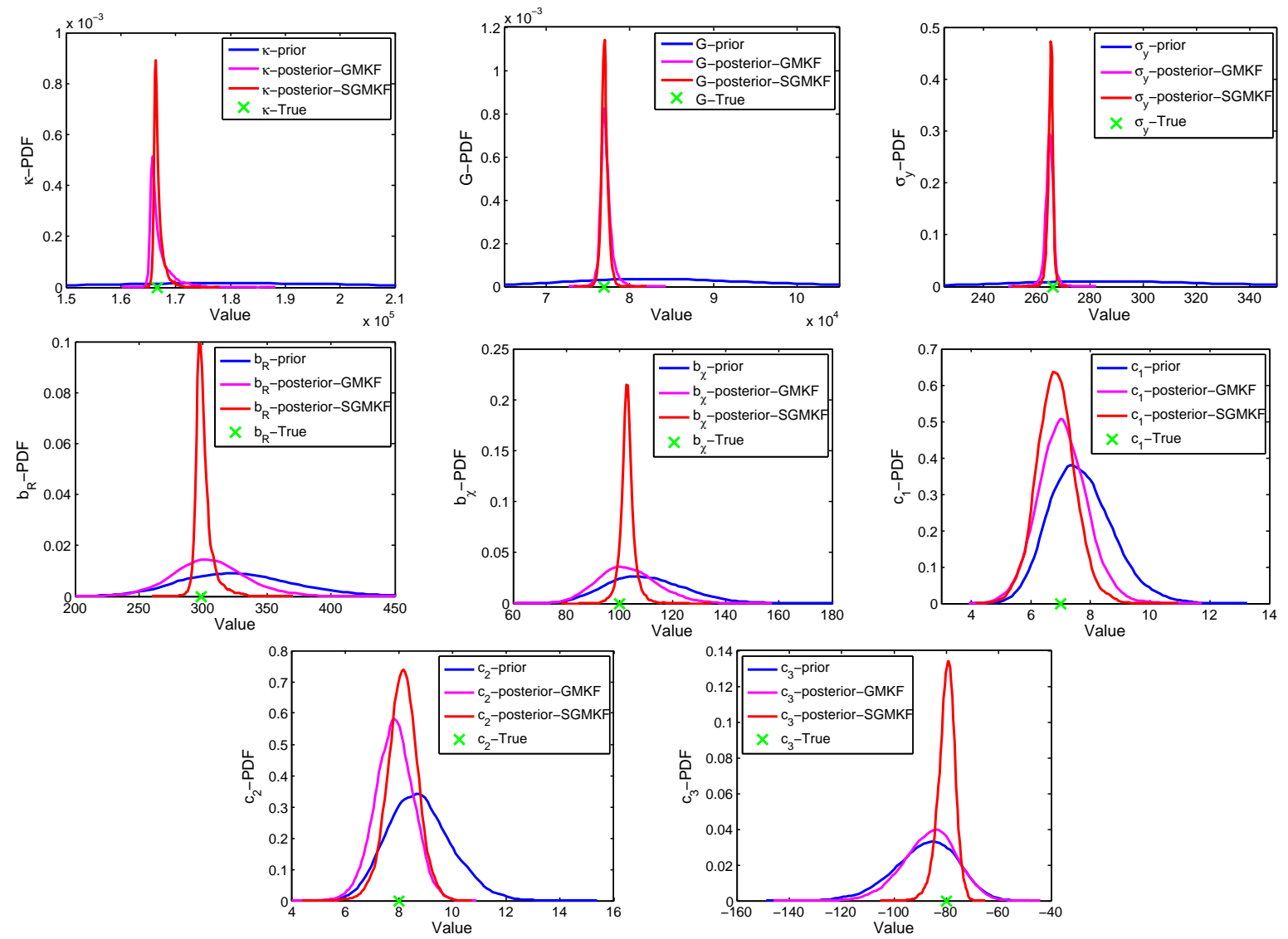

Figure 8. PDF of identified parameters.

From the sharpness of the posterior PDF of $\kappa, G$ and $\sigma_{y}$, it can be concluded that enough information from virtual data is received, and updating the parameters considering their uncertainty is done much easier than the hardening and damage parameters. One reason that can be mentioned 
is that the process is not always in the states that hardening and damage equations are involved. Therefore less information from the whole simulation can be analyzed to estimate the hardening parameters and update their parameters' uncertainties. As the evaluation of hardening and local damage are seen in Figure 9, only in the time intervals [0.8 2.0], [3.5 5.0] and [6.6 8.2], and [0.8 2.0] and [6.6 8.2] hardening equations and damage equations are involved respectively. Therefore hardening and damage parameters are updated, but not as much as the rest of the parameters.
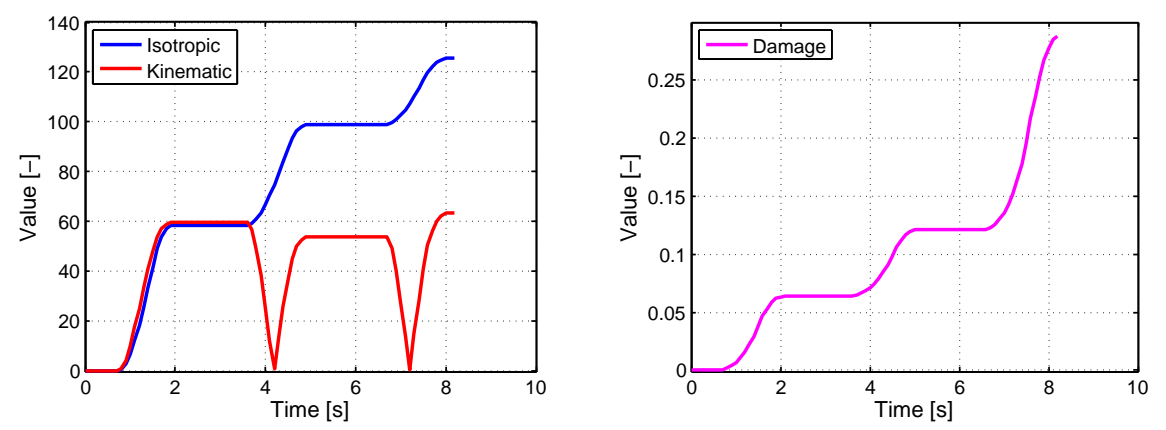

Figure 9. Isotropic and kinematic hardenings- and local damage evaluation according to time [s].

Summarizing the results, the true values and the mean and variance of the estimated parameters are compared in Table 5.

Table 5. The identified model parameters.

\begin{tabular}{lccccc}
\hline Param. & $q_{\text {true }}$ & $q_{\text {GMKF }}^{m}$ & $q_{\text {GMKF }}^{\text {std }}$ & $q_{\text {SGMKF }}^{m}$ & $q_{\text {SGMKF }}^{\text {std }}$ \\
\hline$\kappa$ & $1.66 \times 10^{5}$ & $1.66 \times 10^{5}$ & $2.03 \times 10^{3}$ & $1.66 \times 10^{5}$ & 937.87 \\
$G$ & $7.69 \times 10^{4}$ & $7.70 \times 10^{4}$ & 710.34 & $7.70 \times 10^{4}$ & 430.05 \\
$\sigma_{y}$ & 266 & 264.75 & 1.81 & 265.1 & 1.07 \\
$b_{R}$ & 298.6 & 304.75 & 29.02 & 300.35 & 7.06 \\
$b_{\chi}$ & 100 & 103.12 & 11.18 & 102.98 & 2.77 \\
$c_{1}$ & 7 & 7.07 & 0.82 & 6.84 & 0.65 \\
$c_{2}$ & 8 & 7.82 & 0.70 & 8.13 & 0.57 \\
$c_{3}$ & -80 & -86.29 & 10.29 & -79.98 & 3.34 \\
\hline
\end{tabular}

\section{Conclusions}

The updating is possible by Gauss-Markov-Kalman filter approach for the viscoplastic-damage model with isotropic and kinematic hardening as the updating is performed according to the history matching or sequentially updating method. However it should be pointed out that for the parameters such as hardening and damage parameters, which do not have much strong influence on the measured displacement than the rest of parameters, the identification can not be done well as compared to the rest of parameters. Hence a better identification is achieved when the more information is available from the measurement.

Moreover the uncertainty of the parameters is reduced in the such a way that the posterior distribution functions of the hardening and damage parameters are not as narrow as the rest parameters for the history matching and sequential updating methods.

The sequential updating needs more computational time as the updating process is performed several times than the history updating approach. By considering the fact that the computations are done from the initial time step after each update which leads to observe more information as well. On the other hand, the identification and reduction of the parameter uncertainty as inferred from the update of the parameters is more accurate and acceptable for the sequential updating method than the history matching update as it is seen from the posterior densities of hardening and damage parameters by both approaches shown in Figure 8. 


\section{Summary}

Using the stochastic methods explained in Section 3 to identify the model parameters of the Chaboche damaged model indicates that it is possible to identify the model parameters using Transitional Markov Chain Monte Carlo method and Gauss-Markov Kalman filter approach by means of history updating and also sequentially although the mentioned methods provide different results and the conclusions are discussed below.

The computational time of the Transitional Markov Chain Monte Carlo method on the mentioned model is very high and this results in no sense of applying this method in the economical point of view on the complicated material models. The sequential Gauss-Markov-Kalman filter approach for both variety of the considered models is not as fast as the history matching approach as updating is done multiple times. But still the computation time needed is reasonable and acceptable as a stochastic approach to identify the parameters of a PDE system.

A considerable difference in results is observed between the updated random variables of the uncertain parameters for the considered models which are determined by employing the Gauss-Markov-Kalman filter approach. This is because of the fact that the hardening and damage parameters do not have a strong and direct influence as like the bulk modulus, shear modulus and the yield stress which have a dominant influence on the considered measured displacement output data.

The Gauss-Markov-Kalman filter approach in a history matching update approach is not an optimized method to identify the parameters of the viscoplasticity which includes hardening model and viscoplastic-damage model. This is because, the hardening and damage parameters are not identified well and their uncertainties are not properly reduced as updating is done only once. Further, it is also to be noted that the number of observed states where the bulk modulus, shear modulus and yield stress are involved and identifiable, i.e., the number of states where the elasticity and plasticity part are involved and identifiable are more than the number of states where the hardening and damage parameters are involved and identifiable i.e., only plasticity part.

Based on the reliability, accuracy and computational time, the most suitable method among the employed methods is the sequential Gauss-Markov-Kalman filter approach for the viscoplastic model with isotropic and kinematic hardening and also for the viscoplastic-damage model enriched with hardening behavior. The parameters such as hardening parameters, damage parameters, bulk modulus, shear modulus and yield stress are well updated and posterior distributions representing their uncertainties are much narrow for these parameters. Using sequential Gauss-Markov-Kalman filter approach the parameters should be updated at proper time intervals to save time and to reduce cost. Lesser number of updates results in lesser computation time. Hence particular times should be selected to identify the parameters as discussed in Section 4 where the hardening and damage parameters are changing continuously. Considering this fact results in a better parameter identification and better reduction of parameter uncertainties in a very less time.

It should be noted that the damage evolution has been restricted in such a way that non-local damage should not exceed certain value, i.e., only minor damage is considered for the Gauss-Markov-Kalman filter approach on a viscoplastic-damage model to identify its parameters. Obviously the damage parameters are identified better by using the Gauss-Markov-Kalman filter approach when an immense damage is considered, i.e., the measured displacement data is influenced more by a bigger damage and results in better identification of damage parameters from the provided data. Considering the mentioned fact, this study proves that identification of the model and damage parameters are possible using Bayesian methods before the specimen is really damaged and completely collapsed. Such a Bayesian approach can be recommended to be applied for mechanical structure health monitoring [92,93].

Author Contributions: Conceptualization, formal analysis, investigation, methodology, software, validation, visualization, writing — original draft, writing - review and editing have been done by E.A., H.G.M. and D.D. were in charge of supervison, methodolgy, conceptualization and funding acquisition. B.R. and S.R. have helped E.A. in validation and methodology. All authors have read and agreed to the published version of the manuscript. 
Funding: This work is partially funded by the DFG through GRK 2075.

Conflicts of Interest: The authors declare no conflict of interest.

\section{Appendix A. Elasto-Viscoplastic Constitutive Laws}

Neglecting inertia and using infinitesimal strain theory, the mechanics of the investigated structures with elasto-viscoplastic material behavior is described by the

$$
\begin{gathered}
\text { equilibrium }-\nabla \cdot \sigma=\rho g \\
\text { and the strain balance } \boldsymbol{\epsilon}_{e l}+\boldsymbol{\epsilon}_{v p}=\boldsymbol{\epsilon}
\end{gathered}
$$

see Bonet and Wood [94]. The Cauchy stress $\sigma$ is caused by the body force $\rho g$ specified by density $\rho$ and gravity $g$ and the boundary conditions

$$
\begin{gathered}
u=\bar{u} \\
\sigma \cdot n=\bar{t}
\end{gathered}
$$

on the Dirichlet respectively Neumann part. Here the impressed displacement $\bar{u}$ and the impacting surface tension $\bar{t}$ are marked with a bar, the used symbol for a boundary condition. In Equation (A4) the Cauchy stress is mapped to the boundary by the normal vector $n$ pointing outwards with respect to the structural body. Next to the equilibrium (A1) the strain balance (A2) changes into a differential equation as well when the kinematics of the structure is taken into account. The kinematics is described by the Cauchy strain tensor

$$
\boldsymbol{\epsilon}=\frac{1}{2}\left(\nabla \boldsymbol{u}+(\nabla \boldsymbol{u})^{T}\right)
$$

given by the gradient of the displacement field. In the following, the constitutive equations for the elastic and viscoplastic strains, $\boldsymbol{\epsilon}_{e l}$ respectively $\boldsymbol{\epsilon}_{v p}$, linking the governing Equations (A1) and (A2) are specified in detail.

For mild steel under cyclic loading beyond the yield limit taking isotropic-kinematic hardening into account as well as viscoplastic damaging the material behavior is described by a modified Chaboche model [95] introduced by Kowalsky et al. [74]. Here, damage is described by continuum damage mechanics with evolution equation for isotropic ductile damage $D$, defined later, spatially distributed with the differential equation

$$
D=\bar{D}-l_{c}^{2} \nabla^{2} \bar{D}
$$

depending on the internal length $l_{c}$ as model parameter. Equation (A6) introduces the non-local damage variable $\bar{D}$, marked with a bar as well and used in the concept of effective strains and stresses, see Chaboche [96]. By Equation (A6) it is assumed that damage occurs locally but acts non-locally. It is supposed that only the stress is affected by damage while the strain remains unaffected. The elastic behavior is described by the potential

$$
\phi_{e l}\left(\epsilon_{e l}\right)=\frac{\lambda}{2}\left(\operatorname{tr}\left(\epsilon_{e l}\right)\right)^{2}+\mu \epsilon_{e l}: \epsilon_{e l}
$$

of a St. Venant-Kirchhoff material. The second Lamé-coefficients $\mu$ is alternatively named as shear modulus $G$ in the following. Together with the first Lamé-coefficient, the elastic behavior of the material is further described by the bulk modulus $\kappa=\lambda+\frac{2}{3} \mu$, used from this point onwards. With the tensor of elasticity

$$
C=\frac{\partial^{2} \phi_{e l}}{\partial \epsilon_{e l}^{2}}
$$


the rate formulation of the constitutive equation for the elastic part of the effective strains reads:

$$
\dot{\tilde{\boldsymbol{\epsilon}}}_{e l}=C^{-1}: \dot{\tilde{\sigma}}=\frac{1}{1-\bar{D}} C^{-1}: \dot{\sigma}+\frac{\dot{\bar{D}}}{(1-\bar{D})^{2}} C^{-1}: \sigma
$$

\section{Appendix B. Gauss-Markov-Kalman Filter}

Appendix B.1. Conditional Expectation

The conditional expectation is defined on the Hilbert space where the random variables are considered with finite variance as shown in Equation (A10) where the considered sub- $\sigma$-algebra $\mathfrak{B}$ is a subset of the underlying $\sigma$-algebra $\mathfrak{A}$, i.e., $\mathfrak{B} \subset \mathfrak{A}$. It should be noted that the $\sigma$-algebra is basically representing the collection of subsets of $\Omega$ on which statements about their probability can be made. Therefore it has a continuous orthogonal projection $P_{\mathfrak{B}}: \mathcal{S} \rightarrow \mathcal{S}_{\mathfrak{B}}$ from the whole $\sigma$-algebra to sub- $\sigma$-algebra $\mathfrak{B}$ that has the collection of subsets of $\Omega$ based on which their probability is determined $[69,97]$.

$$
\mathcal{S}_{\mathfrak{B}}:=L_{2}(\Omega, \mathfrak{B}, \mathbb{P}):=\left\{r: \Omega \rightarrow \mathbb{R}: \text { r measurable w.r.t. } \mathfrak{B}, \mathbb{E}\left(|r|^{2}\right)<\infty\right\} \subset \mathcal{S}
$$

Thus the conditional expectation (CE) of a random variable $r \in \mathcal{S}$ with respect to a sub- $\sigma$-algebra $\mathfrak{B}$ can be defined by the orthogonal projection as shown in Equation (A11).

$$
\mathbb{E}(r \mid \mathfrak{B}):=P_{\mathfrak{B}}(r) \in \mathcal{S}_{\mathfrak{B}}
$$

The conditional expectation minimizes the squared error as it is an orthogonal expectation as shown in Equation (A12).

$$
\mathbb{E}\left(|r-\mathbb{E}(r \mid \mathfrak{B})|^{2}\right)=\min \left\{\mathbb{E}\left(|r-\tilde{r}|^{2}\right): \tilde{r} \in \mathcal{S}_{\mathfrak{B}}\right\}
$$

Equation (A12) leads to the orthogonality relation $\forall \tilde{r} \in \mathcal{S}_{\mathfrak{B}}: \mathbb{E}(\tilde{r}(r-\mathbb{E}(r \mid \mathfrak{B})))=0$. Also a form of Pythagoras theorem [98] can be considered as shown in Equation (A13).

$$
\mathbb{E}\left(|r|^{2}\right)=\mathbb{E}\left(|r-\mathbb{E}(r \mid \mathfrak{B})|^{2}\right)+\mathbb{E}\left(|\mathbb{E}(r \mid \mathfrak{B})|^{2}\right)
$$

Therefore it can be concluded that conditional expectation is a form of a minimum mean square error estimator. The conditional probability, for instance, the posterior in Bayes's theorem can be characterized by the conditional expectation $[69,99]$. For instance the conditional probability for $A \subset \Omega$, $A \in \mathfrak{B}$ is obtained by Equation (A14) where the random variable $\chi_{A}$ becomes unity if $\omega \in A$ and it vanishes otherwise.

$$
\mathbb{P}(A \mid \mathfrak{B}):=\mathbb{E}\left(\chi_{A} \mid \mathfrak{B}\right)
$$

Based on Equation (A14), if the conditional expectation $\mathbb{E}\left(\chi_{A} \mid \mathfrak{B}\right)$ is known then everything about the conditional probability $\mathbb{P}(A \mid \mathfrak{B})$ is known and thus the posterior density is defined by this. If the distribution of a random variable representing the prior density is characterized by the prior characteristic function then the conditional characteristic function is determined by using the conditional expectation and this characterizes the conditional distribution. If the prior probability was the distribution of some random variable $r$, then it is completely characterized by the prior characteristic function, i.e., in the sense of probability theory, it can be expressed as $\phi_{r}(s):=\mathbb{E}(\exp ($ irs $))$ [70]. To determine the conditional characteristic function $\phi_{r \mid \mathfrak{B}}(s):=\mathbb{E}(\exp ($ irs $) \mid \mathfrak{B})$, conditional expectation is used instead of the unconditional expectation and this completely characterizes the conditional distribution.

For an instance, if a random variable $y$ is the observation and the sub- $\sigma$-algebra $\mathfrak{B}$ is generated from the observation $y$ resulting in $\mathfrak{B}=\sigma(y)$, the information about the observation can be obtained 
only on $\mathfrak{B}=\sigma(y)$ which are some subsets of $\Omega$ [69]. According to the Doob-Dynkin lemma [100,101], the Equation (A15) represents the subspace $\mathcal{S}_{\sigma(y)}$ as the functions of observation.

$$
\mathcal{S}_{\sigma(y)}:=\{r \in \mathcal{S}: r(\omega)=\phi(y(\omega)), \phi \text { measurable }\} \subset \mathcal{S}
$$

It can be inferred from the Equation (A15) that an observation is a function of the observation and where the information from the measurement is lying is represented by the subspace $\mathcal{S}_{\sigma(y)} \subset \mathcal{S}$ [69].

The conditional expectation $\mathbb{E}(r \mid \sigma(y))=\mathbb{E}(r \mid y)$ and the conditional probability $\mathbb{P}(A \mid \sigma(y))=\mathbb{P}(A \mid y)$ are random variables as $y$ is a random variable. If a fixed value $\hat{y} \in \mathcal{Y}$ is observed for the random variable $y$, i.e., an observation is established then the posterior expectation is just a number $\mathbb{E}(r \mid \hat{y}) \in \mathbb{R}$ and the posterior probability is $\mathbb{P}(A \mid \hat{y})=\mathbb{E}\left(\chi_{A} \mid \hat{y}\right)$. Accordingly, Equation (A16) can be determined for some function $\phi_{r}$ from the Equation (A15) which means for each random variable $r$ it is a possibly different function.

$$
\mathbb{E}(r \mid y)=\phi_{r}(y) \text { and } \mathbb{E}(r \mid \hat{y})=\phi_{r}(\hat{y})
$$

Considering Bayes's theorem $[69,70,101-104]$, it can be concluded that if it is possible to compute the conditional expectation with respect to an observation $y$, then the conditional probability with respect to observation $\hat{y}$ can also be computed and this leads to determine the posterior probability as described in Bayes's theorem. Thus, the Bayesian estimation can be done by using the concept of conditional expectation [69-71].

\section{Appendix B.2. Constructing a Posterior Random Variable}

Equation (A17), which is the probabilistic model of the observation is obtained by considering the random variables and the observation equation of the model as discussed in Equation (10) in Section 2 where the mapping $Y$ is observed. This mapping is on the Hilbert space of the random variables with finite variance.

$$
\hat{y}=y+\epsilon=Y(q)+\epsilon
$$

\section{Appendix B.3. Updating Random Variables}

Let's assume a random variable $\boldsymbol{q}_{n} \in \mathscr{Q}$, the next state $\hat{\boldsymbol{q}}_{n+1} \in \mathscr{Q}$ and the measurement $y_{n+1} \in \mathscr{Y}$ as time $t_{n}$ goes to $t_{n+1}$ can be predicted. The conditional expectation of the measurement prediction $y_{n+1}$ calculated based on Equation (A17) is shown in Equation (A18) and the posterior expectation operator is computed by considering the actual observation $\hat{y}_{n+1}$ as shown in Equation (A19).

$$
\begin{gathered}
\mathbb{E}\left(\Psi\left(\boldsymbol{q}_{n+1}\right) \mid \sigma\left(y_{n+1}\right)\right)=\phi_{\Psi}\left(y_{n+1}\right) \\
\mathbb{E}\left(\Psi\left(\boldsymbol{q}_{n+1}\right) \mid \hat{y}_{n+1}\right)=\phi_{\Psi}\left(\hat{y}_{n+1}\right)
\end{gathered}
$$

As the conditional expectation of the posterior is known, all the information of the posterior probability is available [69-71].

It is to be noted that the Equation (A17) requires every time new random variable $\boldsymbol{q}_{n+2}$ to be computed from $t_{n+1}$ to $t_{n+2}$ and this new random variable has the posterior distribution given by the mappings $\phi_{\Psi}\left(\hat{y}_{n+1}\right)$ as shown in Equation (A19). Although there are many random variables which have this posterior density, only one particular distribution should be chosen via the method as discussed in this section.

To simplify the notations, the forecast random variable is considered as $\boldsymbol{q}_{f}=\hat{\boldsymbol{q}}_{n+1}$ and the forecast measurement is considered as $y_{f}=y_{n+1}$. The measurement is represented as $\hat{y}=\hat{y}_{n+1}$. Therefore the update of the forecast random variable $\boldsymbol{q}_{f}$, also called as the assimilated random variable $\boldsymbol{q}_{a}=\boldsymbol{q}_{n+1}$ results in Equation (A20) where $B$ represent a Bayesian update and $\Xi$ represents 
an innovation map which plays the role of a transformation so-called filter i.e., update by filtering the measurement $\hat{y}[70,71]$.

$$
\boldsymbol{q}_{a}=B\left(\boldsymbol{q}_{f}, y_{f}, \hat{y}\right)=\boldsymbol{q}_{f}+\Xi\left(\boldsymbol{q}_{f}, y_{f}, \hat{y}\right)
$$

Appendix B.4. Correcting the Mean

To correct the mean value of the new random variable $\overline{\boldsymbol{q}}_{a}=\mathbb{E}\left(\boldsymbol{q}_{a} \mid \hat{y}\right)$, it should be considered that $\mathbb{E}\left(\boldsymbol{q}_{a} \mid \sigma\left(y_{f}\right)\right)=\phi_{\boldsymbol{q}_{f}}\left(y_{f}\right):=\phi_{\boldsymbol{q}}\left(y_{f}\right)$ is an orthogonal projection $P_{\sigma\left(y_{f}\right)}\left(\boldsymbol{q}_{f}\right)$ from $\mathscr{Q}=\mathcal{Q} \otimes \mathcal{S}$ onto $\mathscr{Q}_{\infty}:=$ $\mathcal{Q} \otimes \mathcal{S}_{\infty}$, where $\mathcal{S}_{\infty}:=\mathcal{S}_{\sigma(y)}=L_{2}\left(\Omega, \sigma\left(y_{f}\right), \mathbb{P}\right)$ by considering the Equations (A11) and (A19) [69]. Therefore the orthogonal decomposition can be shown as in Equation (A21).

$$
\begin{gathered}
\mathscr{Q}=\mathcal{Q} \otimes \mathcal{S}=\mathscr{Q}_{\infty} \oplus \mathscr{Q}_{\infty}^{\perp}=\left(\mathcal{Q} \otimes \mathcal{S}_{\infty}\right) \oplus\left(\mathcal{Q} \otimes \mathcal{S}_{\infty}^{\perp}\right) \\
\boldsymbol{q}_{f}=P_{\sigma\left(y_{f}\right)}\left(\boldsymbol{q}_{f}\right)+\left(I_{\mathscr{Q}}-P_{\sigma\left(y_{f}\right)}\right)\left(\boldsymbol{q}_{f}\right)=\phi_{\boldsymbol{q}}\left(y_{f}\right)+\left(\boldsymbol{q}_{f}-\phi_{\boldsymbol{q}}\left(y_{f}\right)\right)
\end{gathered}
$$

Equation (A23) can be determined as the conditional expectation of the second term in Equation (A22) is zero as this term is representing the projection part of the decomposition, i.e., $\mathbb{E}\left(\boldsymbol{q}_{f}-\phi_{\boldsymbol{q}}\left(y_{f}\right) \mid \sigma\left(y_{f}\right)\right)=P_{\sigma\left(y_{f}\right)}\left(I_{\mathscr{Q}}-P_{\sigma\left(y_{f}\right)}\right)\left(\boldsymbol{q}_{f}\right)=0$ [69-71]. It can be also explained in this way that when the measurement is obtained, the Equation (A22) is changed by fixing the component $\phi_{q}\left(y_{f}\right) \in \mathscr{Q}_{\infty}$ and leaving the orthogonal rest unchanged.

$$
\boldsymbol{q}_{a, 1}=\phi_{\boldsymbol{q}}(\hat{y})+\left(\boldsymbol{q}_{f}-\phi_{\boldsymbol{q}}\left(y_{f}\right)\right)=\boldsymbol{q}_{f}+\left(\phi_{\boldsymbol{q}}(\hat{y})-\phi_{\boldsymbol{q}}\left(y_{f}\right)\right)
$$

The posterior mean as seen in Equation (A24) can be obtained from Equation (A23).

$$
\overline{\boldsymbol{q}}_{a, 1}=\mathbb{E}\left(\boldsymbol{q}_{a, 1} \mid \hat{y}\right)=\phi_{\boldsymbol{q}}(\hat{y})=\mathbb{E}\left(\boldsymbol{q}_{a} \mid \hat{y}\right)
$$

Appendix B.5. Building the Filter

The algorithm to update the random variable with a map $g: \mathcal{Y} \rightarrow \mathcal{Q}$ to approximate $\phi_{q}$ is obtained by inserting the Equation (A17) into Equation (A23) to obtain the Equation (A25).

$$
\boldsymbol{q}_{n+1}=\boldsymbol{q}_{n}+\left(g\left(\hat{y}_{n+1}\right)-g(Y(\boldsymbol{q})+\epsilon)\right)=\boldsymbol{q}_{n}-g(Y(\boldsymbol{q})+\epsilon)+g\left(\hat{y}_{n+1}\right)
$$

It is inferred from the Equation (A25) that the model Equation (A17) is corrected by an innovation term and the Equation (A25) can be named as the filter equation for identifying the extended uncertain parameters of Equation (A17) which is an unbiased filter with the minimum mean square error estimate represented by $\phi(\hat{y})$.

The map $\phi_{\Psi}$ is defined by Equation (A26). It is obtained by introducing $\omega$ ranging over all measurable maps $\omega: \mathcal{Y} \rightarrow \mathcal{Q}$ and by using the Equation (A12) representing the combination of minimization property and the Equation (A13) representing the Doob-Dynkin lemma $[100,101]$.

$$
\left\|\Psi(\boldsymbol{q})-\phi_{\Psi}(y)\right\|_{\mathscr{Q}}^{2}=\min _{\mathscr{D}}\|\Psi(\boldsymbol{q})-\omega(y)\|_{\mathscr{Q}}^{2}=\min _{z \in \mathscr{Q}_{\infty}}\|\Psi(\boldsymbol{q})-z\|_{\mathscr{Q}}^{2}
$$

As $\mathscr{Q}_{\sigma(y)}=\mathscr{Q}_{\infty}$ is $\mathcal{L}$-closed, it can be concluded that $\forall z \in \mathscr{Q}_{\infty}: \mathbb{E}\left(z \otimes\left(\Psi(\boldsymbol{q})-\phi_{\Psi}(y)\right)\right)$ [105]. Hence the random variable $(\Psi(\boldsymbol{q})-\mathscr{\omega}(y))$ is orthogonal in the $\mathcal{L}$-invariant sense to all random variables $z \in \mathscr{Q}_{\infty}$. In other words, the correlation operator of $(\Psi(\boldsymbol{q})-\omega(y))$ is zero [69-71]. It should also be noted that the measurement operator evaluating $y$ need not necessarily be linear in $q$ and hence the optimal map $\phi_{q}(y)$ also need not necessarily be linear in $y$. However the conditional expectation $\mathbb{E}(\boldsymbol{q} \mid y)=P_{\sigma(y)}(\boldsymbol{q})$ is basically an orthogonal projection. 


\section{References}

1. Adeli, E.; Rosić, B.V.; Matthies, H.G.; Reinstädler, S.; Dinkler, D. Comparison of Bayesian Methods on Parameter Identification for a Viscoplastic Model with Damage. J. Comput. Mech. 2019. [CrossRef]

2. Pacheco, C.; Dulikravich, G.S.; Vesenjak, M.; Borovinsek, M.; Duarte, I.M.A.; Jha, R.; Reddy, S.R.; Orlande, H.R.B.; Colaco, M.J. Inverse parameter identification in solid mechanics using Bayesian statistics, response surfaces and minimization. Tech. Mech. 2016, 36, 120-131.

3. Słonski, M. Bayesian identification of elastic parameters in composite laminates applying lamb waves monitoring. In Proceedings of the Fifth ECCOMAS Thematic Conference on Computational Methods in Structural Dynamics and Earthquake Engineering, Crete Island, Greece, 25-27 May 2015.

4. Zhang, E.; Chazot, J.D.; Antoni, J. Parametric identification of elastic modulus of polymeric material in laminated glasses. In Proceedings of the 16th IFAC Symposium on System Identification, The International Federation of Automatic Control, Brussels, Belgium, 11-13 July 2012.

5. Gallina, A.; Ambrozinski, L.; Packo, P.; Pieczonka, L.; Uhl, T.; Staszewski, W.J. Bayesian parameter identification of orthotropic composite materials using lamb waves dispersion curves measurement. J. Vibr. Control 2017, 23, 2656-2671. [CrossRef]

6. Pieczonka, L.; Gallina, A.; Ambrozinski, L.; Packo, P.; Uhl, T.; Staszewski, W.J. Parameters identification of composite materials using Bayesian approach and guided ultrasonic waves. In Proceedings of the ISMA 2016-International Conference on Noise and Vibration Engineering and USD2016, Leuven, Belgium, 19-21 September 2016.

7. Arnst, M.; Ghanem, R.; Soize, C. Identification of Bayesian posteriors for coefficients of chaos expansions. J. Comput. Phys. 2010, 229, 3134-3154. [CrossRef]

8. Rappel, H.; Beex, L.A.A.; Noels, L.; Bordas, S.P.A. Identifying elastoplastic parameters with Bayes' theorem considering double error sources and model uncertainty. J. Prob. Eng. Mech. 2018. [CrossRef]

9. An, D.; Cho, J.; Kim, N.H. Identification of correlated damage parameters under noise and bias using Bayesian inference. Struct. Health Monit. 2011, 11, 293-303. [CrossRef]

10. Hernandez, W.P.; Borges, F.C.L.; Castello, D.A.; Roitman, N.; Magluta, C. Bayesian inference applied on model calibration of a fractional derivative viscoelastic model. In Proceedings of the XVII International Symposium on Dynamic Problems of Mechanics, Natal, Brazil, 22-27 February 2015.

11. Mahnken, R. Identification of material parameters for constitutive equations. In Encyclopedia of Computational Mechanics, 2nd ed.; Part 2. Solids and Structures; John Wiley \& Sons, Ltd.: Hoboken, NJ, USA, 2017; pp. 1-21.

12. Zheng, W.; Yu, Y. Bayesian probabilistic framework for damage identification of steel truss bridges under joint uncertainties. Adv. Civ. Eng. 2013, 1-13. [CrossRef]

13. Nichols, J.M.; Moore, E.Z.; Murphy, K.D. Bayesian identification of a cracked plate using a population-based Markov Chain Monte Carlo method. J. Comput. Struct. 2011, 89, 1323-1332. [CrossRef]

14. Madireddy, S.; Sista, B.; Vemaganti, K. A Bayesian approach to selecting hyperelastic constitutive models of soft tissue. J. Comput. Appl. Math. 2015, 291, 102-122. [CrossRef]

15. Wang, J.; Zabaras, N. A Bayesian inference approach to the inverse heat conduction problem. Int. J. Heat Mass Trans. 2004, 47, 3927-3941. [CrossRef]

16. Oh, C.K.; Beck, J.L.; Yamada, M. Bayesian learning using automatic relevance determination prior with an application to earthquake early warning. J. Eng. Mech. 2008, 134, 1013-1020. [CrossRef]

17. Alvin, K. Finite element model update via Bayesian estimation and minimization of dynamic residuals. Am. Inst. Aeronaut. Astronaut. J. 1997, 135, 879-886. [CrossRef]

18. Marwala, T.; Sibusiso, S. Finite element model updating using Bayesian framework and modal properties. J. Aircraft 2005, 42, 275-278. [CrossRef]

19. Daghia, F.; de Miranda, S.; Ubertini, F.; Viola, E. Estimation of elastic constants of thick laminated plates within a Bayesian framework. J. Compos. Struct. 2007, 80, 461-473. [CrossRef]

20. Abhinav, S.; Manohar, C. Bayesian parameter identification in dynamic state space models using modified measurement equations. Int. J. Non-Linear Mech. 2015, 71, 89-103. [CrossRef]

21. Gogu, C.; Yin, W.; Haftka, R.; Ifju, P.; Molimard, J.; le Riche, R.; Vautrin, A. Bayesian identification of elastic constants in multi-directional laminate from Moire interferometry displacement fields. J. Exp. Mech. 2013, 53, 635-648. [CrossRef] 
22. Gogu, C.; Haftka, R.; Molimard, J.; Vautrin, A. Introduction to the Bayesian approach applied to elastic constants identification. Am. Inst. Aeronaut. Astronaut. J. 2010, 48, 893-903. [CrossRef]

23. Koutsourelakis, P.S. A novel Bayesian strategy for the identification of spatially varying material properties and model validation: An application to static elastography. Int. J. Num. Methods Eng. 2012, 91, 249-268. [CrossRef]

24. Koutsourelakis, P.S. A multi-resolution, non-parametric, Bayesian framework for identification of spatially-varying model parameters. J. Comput. Phys. 2009, 228, 6184-6211. [CrossRef]

25. Fitzenz, D.D.; Jalobeanu, A.; Hickman, S.H. Integrating laboratory creep compaction data with numerical fault models: A Bayesian framework. J. Geophys. Res. Solid Earth 2007, 112, B08410. [CrossRef]

26. Most, T. Identification of the parameters of complex constitutive models: Least squares minimization vs. Bayesian updating. In Reliability and Optimization of Structural Systems; Straub, D., Ed.; CRC Press: Boca Raton, FL, USA, 2010; pp. 119-130.

27. Sarkar, S.; Kosson, D.S.; Mahadevan, S.; Meeussen, J.C.L.; Sloot, H.v.; Arnold, J.R.; Brown, K.G. Bayesian calibration of thermodynamic parameters for geochemical speciation modeling of cementitious materials. J. Cement Concr. Res. 2012, 42, 889-902. [CrossRef]

28. Zhang, E.; Chazot, J.D.; Antoni, J.; Hamdi, M. Bayesian characterization of Young's modulus of viscoelastic materials in laminated structures. J. Sound Vibr. 2013, 332, 3654-3666. [CrossRef]

29. Mehrez, L.; Kassem, E.; Masad, E.; Little, D. Stochastic identification of linear-viscoelastic models of aged and unaged asphalt mixtures. J. Mater. Civ. Eng. 2015, 27, 04014149. [CrossRef]

30. Miles, P.; Hays, M.; Smith, R.; Oates, W. Bayesian uncertainty analysis of finite deformation viscoelasticity. J. Mech. Mater. 2015, 91, 35-49. [CrossRef]

31. Zhao, X.; Pelegri, A.A. A Bayesian approach for characterization of soft tissue viscoelasticity in acoustic radiation force imaging. Int. J. Num. Methods Biomed. Eng. 2016, 32, E02741. [CrossRef] [PubMed]

32. Kenz, Z.R.; Banks, H.T.; Smith, R.C. Comparison of frequentist and Bayesian confidence analysis methods on a viscoelastic stenosis model. SIAM/ASA J. Uncertain. Quant. 2013, 1, 348-369. [CrossRef]

33. An, D.; Choi, J.; Kim, N.H.; Pattabhiraman, S. Fatigue life prediction based on Bayesian approach to incorporate field data into probability model. J. Struct. Eng. Mech. 2011, 37, 427-442. [CrossRef]

34. Hoshi, T.; Kobayashi, Y.; Kawamura, K.; Fujie, M.G. Developing an intraoperative methodology using the finite element method and the extended Kalman filter to identify the material parameters of an organ model. In Proceedings of the 29th Annual International Conference of the IEEE EMBS Cite Internationale, Lyon, France, 23-26 August 2007.

35. Furukawa, T.; Pan, J.W. Stochastic identification of elastic constants for anisotropic materials. Int. J. Num. Methods Eng. 2010, 81, 429-452. [CrossRef]

36. Conte, J.P.; Astroza, R.; Ebrahimian, H. Bayesian methods for nonlinear system identification of civil structures. MATEC Web Conf. 2015, 24, 03002. [CrossRef]

37. Hendriks, M.A.N. Identification of the Mechanical Behavior of Solid Materials. Ph.D. Thesis, Department of Mechanical Engineering, Technische Universiteit Eindhoven, Eindhoven, The Netherlands, 1991.

38. Bolzon, G.; Fedele, R.; Maier, G. Parameter identification of a cohesive crack model by Kalman filter. J. Comput. Methods Appl. Mech. Eng. 2002, 191, 2847-2871. [CrossRef]

39. Astroza, R.; Nguyen, L.T.; Nestorović, T. Finite element model updating using simulated annealing hybridized with unscented Kalman filter. J. Comput. Struct. 2016, 177, 176-191. [CrossRef]

40. Mahmoudi, E.; König, M.; Schanz, T. Probabilistic analysis of a gas storage cavity mined in a spatially random rock salt medium. In Proceedings of the Coupled Problems 2017, Rhodes, Greece, 12-14 June 2017.

41. Mahmoudi, E.; Khaledi, K.; König, D.; Schanz, T. Probabilistic analysis of a rock salt cavern with application to energy storage systems, using subset simulation methodology. In Energy Geotechnics; Wuttke, F., Bauer, S., Sanchez, M., Eds.; CRC Press: Boca Rotan, FL, USA, 2016; pp. 609-615.

42. Wall, O.; Holst, J. Estimation of parameters in viscoplastic and creep material models. SIAM J. Appl. Math. 2001, 61, 2080-2103. [CrossRef]

43. Nakamura, T.; Gu, Y. Identification of elastic-plastic anisotropic parameters using instrumented indentation and inverse analysis. J. Mech. Mater. 2007, 39, 340-356. [CrossRef]

44. Agmell, M.; Ahadi, A.; Stahl, J. Identification of plasticity constants from orthogonal cutting and inverse analysis. J. Mech. Mater. 2014, 77, 43-51. [CrossRef] 
45. Sevieri, G.; Andreini, M.; Falco, A.D.; Matthies, H.G. Concrete gravity dams model parameters updating using static measurements. Eng. Struct. 2019, 196. [CrossRef]

46. Sevieri, G.; Falco, A.D. Dynamic structural health monitoring for concrete gravity dams based on the Bayesian inference. J. Civil Struct. Health Monit. 2020, 10, 235-250. [CrossRef]

47. Sevieri, G.; Falco, A.D.; Marmo, G. Shedding Light on the Effect of Uncertainties in the Seismic Fragility Analysis of Existing Concrete Dams. Infrastructures 2020, 5, 22. [CrossRef]

48. Marsili, F.; Croce, P.; Friedman, N.; Formichi, P.; Landi, F. Seismic reliability assessment of a concrete water tank based on the Bayesian updating of the finite element model. ASCE-ASME J. Risk Uncertain. Eng. Syst. Part B Mech. Eng. 2017, 3, 021004. [CrossRef]

49. Marsili, F.; Croce, P.; Friedman, N.; Formichi, P.; Landi, F. On Bayesian identification methods for the analysis of existing structures. In Proceedings of the IABSE Congress Stockholm, Challenges in Design and Construction of an Innovative and Sustainable Built Environment, Stockholm, Sweden, 21-23 September 2016; pp. 116-123.

50. Croce, P.; Landi, F.; Formichi, P. Probabilistic Seismic Assessment of Existing Masonry Buildings. Buildings 2019, 9, 237. [CrossRef]

51. Croce, P.; Formichi, P.; Landi, F. A Bayesian hierarchical model for climatic loads under climate change. Eccomas Proc. UNCECOMP 2019, 298-308. [CrossRef]

52. Croce, P.; Marsili, F.; Klawonn, F.; Formichi, P.; Landi, F. Evaluation of statistical parameters of concrete strength from secondary experimental test data. Constr. Build. Mater. 2018, 163, 343-359, doi:10.1016/j.conbuildmat.2017.11.001. [CrossRef]

53. Bocciarelli, M.; Bolzon, G.; Maier, G. A constitutive model of metal-ceramic functionally graded material behavior: Formulation and parameter identification. J. Comput. Mater. Sci. 2008, 43, 16-26. [CrossRef]

54. Gu, Y.; Nakamura, T.; Prchlik, L.; Sampath, S.; Wallace, J. Micro-indentation and inverse analysis to characterize elastic-plastic graded materials. J. Mater. Sci. Eng. A 2003, 345, 223-233. [CrossRef]

55. Corigliano, A.; Mariani, S. Parameter identification of a time-dependent elastic-damage interface model for the simulation of debonding in composites. J. Compos. Sci. Technol. 2001, 61, 191-203. [CrossRef]

56. Corigliano, A.; Mariani, S. Simulation of damage in composites by means of interface models: Parameter identification. J. Compos. Sci. Technol. 2001, 61, 2299-2315. [CrossRef]

57. Ebrahimian, H.; Astroza, R.; Conte, J.P.; de Callafon, R.A. Nonlinear finite element model updating for damage identification of civil structures using batch Bayesian estimation. J. Mech. Syst. Signal Process. 2017, 84, 194-222. [CrossRef]

58. Ebrahimian, H.; Astroza, R.; Conte, J.P. Parametric Identification of Hysteretic Material Constitutive Laws in Nonlinear Finite Element Models using Extended Kalman Filter; Department of Structural Engineering, University of California: San Diego, CA, USA, 2014.

59. Ebrahimian, H.; Astroza, R.; Conte, J.P. Extended Kalman filter for material parameter estimation in nonlinear structural finite element models using direct differentiation method. Earthq. Eng. Struct. Dyn. 2015, 44, 1495-1522. [CrossRef]

60. Astroza, R.; Ebrahimian, H.; Conte, J.P. Material parameter identification in distributed plasticity FE models of frame-type structures using nonlinear stochastic filtering. J. Eng. Mech. 2015, 141, 04014149. [CrossRef]

61. Hariri-Ardebili, M.A.; Heshmati, M.; Boodagh, P.; Salamon, J.W. Probabilistic Identification of Seismic Response Mechanism in a Class of Similar Arch Dams. Infrastructures 2019, 4, 44. [CrossRef]

62. Saouma, V.E.; Hariri-Ardebili, M.A. Probabilistic Cracking, Aging and Shaking of Concrete Dams. In Proceedings of the 5th International Symposiumon Dam Safety, Istanbul, Turkey, 27-31 October 2018; pp. 44-56.

63. Yan, A.; de Boe, P.; Golinval, J. Structural damage diagnosis by Kalman model based on stochastic subspace identification. Int. J. Struct. Health Monit. 2004, 3, 103-119. [CrossRef]

64. Ghannadi, P.; Kourehli, S.S. Model updating and damage detection in multi-story shear frames using salp swarm algorithm. Earthq. Struct. 2019, 17, 63-73.

65. Ghannadi, P.; Kourehli, S.S.; Noori, M.; Altabey, W.A. Efficiency of grey wolf optimization algorithm for damage detection of skeletal structures via expanded mode shapes. Adv. Struct. Eng. 2020. [CrossRef]

66. Gharehbaghi, V.; Nguyen, A.; Farsangi, E.N.; Yang, T.Y. Supervised damage and deterioration detection in building structures using an enhanced autoregressive time-series approach. J. Build. Eng. 2020. [CrossRef] 
67. Ching, J.; Chen, Y.C. Transitional Markov Chain Monte Carlo method for Bayesian model updating, model class selection and model averaging. J. Eng. Mech. 2007, 133, 816-832. [CrossRef]

68. Ching, J.; Wang, J. Application of the Transitional Markov Chain Monte Carlo algorithm to probabilistic site characterization. J. Eng. Geol. 2016, 203, 151-167. [CrossRef]

69. Matthies, H.G.; Zander, E.; Rosić, B.V.; Litvinenko, A.; Pajonk, O. Inverse problems in a Bayesian setting. J. Comput. Methods Solids Fluids 2016, 245-286.

70. Matthies, H.G.; Zander, E.; Rosić, B.V.; Litvinenko, A. Parameter estimation via conditional expectation: A Bayesian inversion. Adv. Model. Simulat. Eng. Sci. 2016, 3, 24. [CrossRef]

71. Matthies, H.G.; Litvinenko, A.; Rosić, B.V.; Zander, E. Bayesian parameter estimation via filtering and functional approximations. Tech. Gazette 2016, 23, 1-8.

72. Pajonk, O.; Rosić, B.V.; Litvinenko, A.; Matthies, H.G. A deterministic filter for non-Gaussian Bayesian estimation-Applications to dynamical system estimation with noisy measurements. Phys. D Nonlinear Phenomena 2012, 241, 775-788. [CrossRef]

73. Simo, J.C.; Hughes, T.J.R. Computational Inelasticity, 7th ed.; Springer: New York, NY, USA, 1998.

74. Kowalsky, U.; Meyer, J.; Heinrich, S.; Dinkler, D. A nonlocal damage model for mild steel under inelastic cyclic straining. Comput. Mater. Sci. 2012, 63, 28-34. [CrossRef]

75. Pirondi, A.; Bonora, N. Modeling ductile damage under fully reversed cycling. Comput. Mater. Sci. 2003, 26, 129-141. [CrossRef]

76. Hughes, T.; Hulbert, G. Space-time finite element methods for elastodynamics: Formulations and error estimates. Comput. Methods Appl. Mech. Eng. 1988, 66, 339-363. [CrossRef]

77. Zienkiewicz, O.C.; Taylor, R.L.; Zhu, J.Z. The Finite Element Method: Its Basis and Fundamentals, 6th ed.; Elsevier Butterworth-Heinemann: Oxford, UK, 2005; ISBN 978-1-85617-633-0.

78. Wang, J.; Katafygiotis, L.S.; Noori, M.N. Transitional Markov Chain Monte Carlo simulation for reliability-based optimization. In Safety, Reliability, Risk and Life-Cycle Performance of Structures \& Infrastructures; Deodatis, G., Ellingwood, B.R., Frangopol, D.M., Eds.; CRC Press: Boca Raton, FL, USA, 2014; pp. 1593-1599.

79. McGrayne, S.B. The Theory That Would Not Die; Yale University Press: New Haven, CT, USA, 2011.

80. Luenberger, D.G. Optimization by Vector Space Methods; Wiley: Chichester, UK, 1969.

81. Grewal, M.S.; Andrews, A.P. Kalman Filtering: Theory and Practice Using MATLAB; Wiley: Chichester, UK, 2008.

82. Evensen, G. Data Assimilation-The Ensemble Kalman Filter; Springer: Berlin/Heidelberg, Germany, 2009.

83. Pajonk, O. Stochastic Spectral Methods for Linear Bayesian Inference. Ph.D. Thesis, Institut für Wissenschaftliches Rechnen, Technische Universität Braunschweig, Braunschweig, Germany, 2012.

84. Matthies, H.G. Stochastic finite elements: Computational Approaches to Stochastic Partial Differential Equations. J. Appl. Math. Mech. 2008, 88, 849-873. [CrossRef]

85. Xiu, D. Numerical Methods for Stochastic Computations: A Spectral Method Approach; Princeton University Press: Princeton, NJ, USA, 2010.

86. Wiener, N. The homogeneous chaos. Am. J. Math. 1938, 60, 897-936. [CrossRef]

87. Ghanem, R.; Spanos, P.D. Stochastic Finite Eements-A Spectral Approach; Springer: Berlin/Heidelberg, Germany, 1991.

88. Adeli, E.; Rosić, B.V.; Matthies, H.G.; Reinstädler, S. Bayesian parameter identification in plasticity. In Proceedings of the XIV International Conference on Computational Plasticity, Fundamentals and Applications COMPLAS XIV, Barcelona, Spain, 5-7 September 2017.

89. Adeli, E.; Rosić, B.V.; Matthies, H.G.; Reinstädler, S. Effect of Load Path on Parameter Identification for Plasticity Models using Bayesian Methods. In Lecture Notes in Computational Science and Engineering; Nature Springer: 2018; Available online: http:/ /arxiv.org/abs/1906.07246 (accessed on 17 June 2019).

90. Adeli, E.; Matthies, H.G. Parameter Identification in Viscoplasticity using Transitional Markov Chain Monte Carlo Method. Available online: http:/ / arxiv.org/abs/1906.10647 (accessed on 21 June 2019).

91. Felippa, C. Introduction to FEM, FEM Modeling: Mesh, Loads and BCs; University of Colorado: Boulder, CO, USA, 2016.

92. Adeli, E. Viscoplastic-Damage Model Parameter Identification via Bayesian Methods. Ph.D. Dissertation, Institut für Wissenschaftliches Rechnen, Technische Universität Braunschweig, Braunschweig, Germany, 2019. [CrossRef] 
93. Adeli, E.; Rosić, B.V.; Matthies, H.G.; Reinstädler, S.; Dinkler, D. Bayesian Parameter Determination of a CT-Test Described by a Viscoplastic-Damage Model Considering the Model Error. 2019. Available online: https:/ / www.researchgate.net/publication/334537857_Bayesian_Parameter_Determination_of_a_ CT-Test_described_by_a_Viscoplastic-Damage_Model_considering_the_Model_Error (accessed on 21 June 2019).

94. Bonet, J.; Wood, R.D. Nonlinear Continuum Mechanics for Finite Element Analysis; Cambridge University Press: Cambridge, UK, 1997; ISBN 978-0521838702.

95. Chaboche, J.L.; Rousselier, G. On the plastic and viscoplastic constitutive equations-Part 1: Rules developed with internal variable concept. J. Press. Vessel Technol. 1983, 105, 153-158. [CrossRef]

96. Chaboche, J.L.; Rousselier, G. On the plastic and viscoplastic constitutive equations-Part 2: Application of internal variable concepts to the 316 stainless steel. J. Press. Vessel Technol. 1983, 105, 159-164. [CrossRef]

97. Bobrowski, A. Functional Analysis for Probability and Stochastic Processes; Cambridge University Press: Cambridge, UK, 2005.

98. Pritchard, C. The Changing Shape of Geomtetry; Cambridge University Press: Cambridge, UK, 2003.

99. Rao, M. Conditional Measures and Applications; CRC Press: Boca Raton, FL, USA, 2005.

100. Rao, M.; Swift, R.J. Probability Theory with Applications. Mathematics and Its Applications, Band 582; Springer: Berlin/Heidelberg, Germany, 2006.

101. Tarantola, A. Inverse Problem Theory and Methods for Model Parameter Estimation; SIAM: Philadelphia, PA, USA, 2004.

102. Jaynes, E.T. Probability Theory, the Logic of Science; Cambridge University Press: Cambridge, UK, 2003.

103. Aster, R.C.; Borchers, B.; Thurber, C.H. Parameter Estimation and Inverse Problems, 2nd ed.; Academic Press: Cambridge, MA, USA, 2012; ISBN 978-0-12-804651-7.

104. Dashti, M.; Stuart, A.M. The Bayesian Approach to Inverse Problems. In Handbook of Uncertainty Quantification; Springer Nature: Cham, Switzerland, 2015; pp. 1-118.

105. Bosq, D. Linear processes in function spaces, theory and applications. In Lecture Notes in Statistics 149; Springer: Berlin/Heidelberg, Germany, 2000.

(C) 2020 by the authors. Licensee MDPI, Basel, Switzerland. This article is an open access article distributed under the terms and conditions of the Creative Commons Attribution (CC BY) license (http://creativecommons.org/licenses/by/4.0/). 